\title{
PERFORMANCE OF A SCINTILLATING FIBRE DETECTOR FOR THE UA2 UPGRADE
}

\author{
R.E. Ansorge 1), C. Aurouet 2), P. Bareyre 2), P. Bonamy 2), C.N. Booth 3), M. Bouchard 2), \\ M. Bourdinaud 2), M. Cordier 2), J. Crittenden 2), J. Dupont 3), J. Dupraz 3), \\ K. Einsweiler 3), C. Engster 3), J-P. Fabre 3), J-M. Gaillard 4), J-L. Garavel 3), \\ K. Hultqvist 3), C. Jeanney 2), S.G. Katvars 1), M. Lefebvre 1), L. Linssen 3), \\ B. McCluskey 1), B. Merkel 4), J-P. Meyer 2), D.J. Munday 1), P. Nappey 3), J.M. Pentney 1), \\ S. Reynaud 3), J.G. Rushbrooke 1), J-C. Thevenin 2), W.Y. Tsang D), A.R. Weidberg 3), \\ P.S. Wells 1), S.A. Wotton 1), T.O. White 1) and H. Zaccone 2)
}

\begin{abstract}
A large scintillating fibre detector for the UA2 experiment at the CERN pp Collider is under construction. It will be used for tracking and electron identification. The performance of a full scale test module containing 960 fibres of $2.1 \mathrm{~m}$ length and $1 \mathrm{~mm}$ diameter is described.
\end{abstract}

Invited contribution to the International Conference on Advances in Experimental Methods for Colliding Beam Physics, SLAC, 9-13 March 1987.

1) Cavendish Laboratory, University of Cambridge, Cambridge, United Kingdom

2) Centre d'Etudes Nucléaires de Saclay, 91191 Gif-sur-Yvette, France

3) CERN, 1211 Geneva 23, Switzerland

4) Laboratoire de 1'Accélérateur Linéaire, Université Paris-Sud, Orsay, France 


\section{INTRODUCTION}

The upgrade of the UA2 experiment at CERN [1] is designed to take full advantage of the increased luminosity that will be available at the SPS Collider after completion of the Antiproton Collector (ACOL) [2] at the end of 1987. One of the main physics motivations will be the search for electrons with $\mathrm{p}_{\mathrm{T}}$ in the range $10-30 \mathrm{GeV} / \mathrm{c}$ as a signature for top quark production. This will require improved electron identification which will be provided by the new cylindrical vertex detector, which fits inside the existing central calorimeter. Apart from a jet vertex detector and a layer of silicon pads it will include a transition radiation detector, which fills half of the available radial space, implying the need for a very compact tracking and preshower detector. This is achieved using a Scintillating Fibre Detector (SFD).

The SFD is a $2.1 \mathrm{~m}$ long cylindrical detector consisting of 24 layers of $1 \mathrm{~mm}$ diameter scintillating plastic fibres ( 60000 fibres in total). Its inner radius is $388 \mathrm{~mm}$ and it extends close to the central calorimeter at a radius of $440 \mathrm{~mm}$. It covers polar angles from $20^{\circ}$ to $160^{\circ}$. The layers are arranged in 8 stereo triplets. The inner 6 triplets are used as a tracking detector. They are followed by a 1.5 radiation length lead converter in the region of the central calorimeter in order to use the last two triplets as a preshower detector. In each stereo triplet the angles of the fibres with respect to the axis of the cylinder are $-\alpha, 0$, and $\alpha$, with $\alpha=15.75^{\circ}$ for the tracking part and $\alpha=21^{\circ}$ for the preshower part. This paper describes results of beam tests of an SFD prototype.

\section{THE TEST SETUP}

A full scale setup consisting of 960 scintillating plastic fibres and one complete read-out chain was tested with $40 \mathrm{GeV}_{\pi^{-}}$s, $10 \mathrm{GeV}$ and $40 \mathrm{GeV} \mathrm{e}^{-' s}$ and $\sim 160 \mathrm{GeV} \mu^{-\prime}$ s using the $\mathrm{H} 2$ beam of the SPS.

The passage of a charged particle is registered by a coincidence of the signals from three scintillation counters placed in the beam-line and its position is measured in two multiwire chambers with two-dimensional read-out at distances of $1.5 \mathrm{~m}$ behind and $4.4 \mathrm{~m}$ in front of the fibre stack. The pion contamination in the electron beam was $\sim 1 \%$ and the electron contamination in the pion beam was less than $\sim 0.5 \%$. The beam intensity was chosen to be typically $\sim 4000$ per burst of $\sim 2$ sec for muons and $500-1500$ per burst for pions and electrons.

\subsection{The Fibres}

The fibres consist of a $1 \mathrm{~mm}$ core of polystyrene doped with butyl-PBD and POPOP, which produces wavelength-shifted scintillation light of about $440 \mathrm{~nm}$. The core, which has refractive index 1.59 , is surrounded with a $15 \mu \mathrm{m}$ cladding of refractive index 1.46 . The cladding is necessary to preserve the optical quality of the scintillator surface and to ensure total internal reflection for the light striking the core-cladding interface at incident angles greater than the critical angle of $67^{\circ}$ which represents typically $8 \%$ of the total amount of scintillation light produced. To prevent cross-talk between adjacent fibres, the fibres are aluminised by sputtering. The $0.1 \mu \mathrm{m}$ aluminium layer has been shown to have no detrimental effect on the optical properties and also improves the mechanical stability of the fibres. The light from each fibre is collected at one end only. The attenuation of the light, $\lambda_{\text {att }} \sim 1.6 \mathrm{~m}$, is partly compensated by a reflective sheet of aluminised mylar at the opposite end. This means of reflection will be replaced in the definitive version of the detector by a sputtered aluminium coating, improving the coefficient of reflection from about 0.3 to 0.6 . The light yield at a distance of $1.3 \mathrm{~m}$ for a minimum ionising particle passing through $1 \mathrm{~mm}$ of fibre is $\sim 30$ photons. The total length of the fibres is $2.35 \mathrm{~m}$.

The fibre bundle used for the tests consists of 24 layers of 40 contiguous fibres fixed on adhesive tape (see fig. 1). For convenience all fibres in the bundle have a parallel orientation (no stereo angles), but as in the final detector, groups of three layers of fibres are separated by $1.5 \mathrm{~mm}$ rohacell foam lay- 
ers. After the first 18 layers a lead converter is inserted, allowing the use of the last two triplets as a preshower counter. Apart from the normal beam impact at half-length of the fibres, tracks leaving the $\overline{\mathrm{p} p}$ interaction vertex at $\theta=28^{\circ}, 45^{\circ}, 135^{\circ}$ and $152^{\circ}$ are simulated by rotating the fibre bundle in the test bearm around the imaginary interaction point at $42 \mathrm{~cm}$ distance from the bundle. At normal beam incidence the lead converter has a thickness of $8.4 \mathrm{~mm}$; at the $45^{\circ}$ and $135^{\circ}$ positions it is reduced to $5.9 \mathrm{~mm}$ such that the particles traverse 1.5 radiation lengths independently of their angle. There is no converter at $\theta=28^{\circ}$ and $\theta=152^{\circ}$. The total thickness of the fibre stack amounts to $52 \mathrm{~mm}$; its active length is $2.1 \mathrm{~m}$. The additional fibre length of $25 \mathrm{~cm}$ is used for coupling to the read-out. A group of 45 fibres, uniformly distributed throughout the stack, can be illuminated through slits in the reflective sheet at the end of the fibre opposite to the read-out and serve as a fiducial system.

\subsection{The Read-Out and Data Acquisition}

The light output of all 960 fibres is amplified, converted into an electric signal, multiplexed and digitised by a single read-out chain. The fibre ends are grouped in a non-compact rectangular array by a perforated plate and optically coupled to the input of an image intensifier chain. The minimum distance between fibre-centres in the plate is $1.3 \mathrm{~mm}$. The 960 fibres of the prototype detector fill only half of the coupling plate, which will contain $\sim 2000$ fibres in the final detector. The image intensifier chain consists of three electrostatic tubes, of which one is equipped with a micro channel plate. They serve to both demagnify and amplify the light signals from the fibres. The light output from the image intensifiers is subsequently detected and multiplexed in a charge coupled device (CCD). The output from the CCD is digitised by a purpose-built FASTBUS digitiser, which applies substantial data reduction. The digitiser information is monitored and transmitted to a VAX-750 computer via CAMAC by a FASTBUS processor, the Aleph event builder [3].

\subsubsection{The Image Intensifier System}

The image intensifier system (see fig. 1) consists of two electrostatically focused image intensifiers (II) and one proximity focused II containing a micro channel plate (MCP). Photons entering an II strike a photocathode and liberate electrons. These electrons are accelerated by a strong electric field and hit a phosphor screen, where each energetic electron liberates a number of photons. The first II reduces the image of the fibres from $80 \mathrm{~mm}$ diameter to $16 \mathrm{~mm}$. It has a light amplification of $\sim 10$. Its cathode-anode voltage is $20 \mathrm{kV}$ and it contains two additional electrodes to zoom and focus the image. The second II has a diameter of $18 \mathrm{~mm}$. It preserves the size of the image, but has a much larger light amplification of $\sim 800$ as it contains a micro channel plate (MCP). The MCP consists of a $0.4 \mathrm{~mm}$ thick, $18 \mathrm{~mm}$ diameter array of close-packed $12 \mu \mathrm{m}$ thin hollow glass tubes across which a $650 \mathrm{~V}$ voltage difference is applied. The electrons, which are produced at the II photocathode, are accelerated over a $200 \mathrm{~V}$ potential difference and enter the MCP, where electron multiplication takes place in successive interactions between the electrons and the tube walls. The electrons leaving the MCP are accelerated by $6 \mathrm{kV}$ across a short gap before they strike the phosphor screen and produce light. The third II reduces the $16 \mathrm{~mm}$ image to a $6 \mathrm{~mm}$ diameter image which matches the size of the CCD. It has a cathode-anode voltage difference of $15 \mathrm{kV}$ and a light amplification of $\sim 6$. All the image intensifiers are manufactured by $\mathrm{DEP}^{1}$. The parameters of the image intensifiers are summarised in table 1 . To preserve the optical resolution of the image all $\mathrm{II}^{\prime}$ s are equipped with fibre-optic entry and exit windows consisting of $6 \mu \mathrm{m}$ diameter fibres. Fast phosphor screens are required as the light output from one event must have decayed to zero before the next event may occur $(3.8 \mu \mathrm{s}$ later) at the $\bar{p} p$ Collider. The first two II's are equipped with blue P47 phosphors [4] (decay time $\sim 50 \mathrm{~ns}$ ) to match the S20 photocathodes. The third II has a green P46 phosphor [4] (decay time $\sim 100 \mathrm{~ns}$ ) to match the light sensitivity of the CCD. All II's have S20 photocathodes, with quantum efficiencies of $13 \%-16 \%$ (see table 1). The average number of photoelectrons produced at the first photocathode per minimum ionising particle traversing $1 \mathrm{~mm}$ of fibre at $\theta=90^{\circ}$ is $\sim 3.6$ (see section 3.5). The total amplification loss due to the coupling between the image intensifiers is $\sim 1.5$. The second II is gated off $2 \mu \mathrm{s}$ after receiving a trigger to prevent light output from the II chain during the image transfer in the CCD. This

\footnotetext{
${ }^{1}$ Delft Electronische Producten, Roden, The Netherlands.
} 
is done by making the MCP entrance voltage more negative than the photocathode potential. The effective integration time of $2 \mu \mathrm{s}$ is long compared to the phosphor decay time but short enough to keep II noise negligible.

\begin{tabular}{|c|c|c|c|}
\hline & $\mathrm{II}_{1}$ & $\mathrm{II}_{2}$ & $\mathrm{II}_{3}$ \\
\hline input diameter & $80 \mathrm{~mm}$ & $18 \mathrm{~mm}$ & $18 \mathrm{~mm}$ \\
\hline output diameter & $18 \mathrm{~mm}$ & $18 \mathrm{~mm}$ & $7 \mathrm{~mm}$ \\
\hline photon gain & $\sim 10$ & $\sim 800$ & $\sim 6$ \\
\hline cathode-anode potential & $20 \mathrm{kV}$ & $7 \mathrm{kV}$ & $15 \mathrm{kV}$ \\
\hline photocathode & $\mathrm{S} 20$ & $\mathbf{S} 20$ & $\mathbf{S} 20$ \\
\hline quantum efficiency & $>13 \%$ & $16 \%$ & $16 \%$ \\
\hline phosphor & $\mathrm{P} 47$ & P47 & P46 \\
\hline
\end{tabular}

The gain of the MCP is known to decrease with total integrated current. We have measured this ageing effect and estimate that a decay in gain of $1 / \mathrm{e}$ will occur after an integrated luminosity of 13 in verse picobarns at the Collider (see Appendix). This loss in gain may be compensated by gradually increasing the voltage across the MCP, which has a safety margin of $\sim 300 \mathrm{~V}$, corresponding to a gain increase of a factor 28. The average light-output from the fibres in the preshower part of the detector will be approximately 3-4 times larger than in the tracking part, as electrons and photons will produce showers containing many charged particles. To match the ageing effects in the MCP and to avoid saturation in the CCD electronics a light attenuator is placed between the first and second image intensifier. This attenuator consists of a transparent mylar sheet with an $\sim 80 \AA$ thick chromium deposit, which reduces the light from the preshower region by a factor of 4

\subsubsection{CCD Operation}

A $\mathrm{CCD}^{2}$ is used to measure the light output from the image intensifiers. The CCD is an array of about 32000 photosensitive detectors (pixels) grouped in a single silicon substrate. Cross sections through the CCD are shown schematically in fig. 2. By applying suitable voltages to the "clock" electrodes $\Phi_{1 p}$ and $\Phi_{2 p}$ an array of potential wells is created in the silicon substrate. Photons striking the $C C D$ produce electron-hole pairs and the resulting electrons are trapped in these potential wells. The CCD consists of an image zone of 145 lines each consisting of 218 pixels. In each line 208 pixels are photosensitive, the remaining pixels are used to measure the dark image level. Each pixel has a size of $30 \mu \mathrm{m}$ by $28 \mu \mathrm{m}$. There is also a non-photosensitive memory zone of the same size (see fig.3). Through manipulation of the clock voltages the charge in one line can be shifted to the next (charge transfer).

For the operation at the Collider it will be essential to clear the image of an unwanted event before the next bunch crossing ( $3.8 \mu \mathrm{s}$ later). A fast clear of the image zone can be achieved in $1 \mu \mathrm{s}$ using the anti-blooming electrodes. The principle of the anti-blooming electrodes is shown in fig. $2 \mathrm{c}$. In the conventional use of CCDs the antiblooming facility is used to prevent the light from a bright spot on the image spreading out into neighbouring parts of the image ("blooming"). If there is too much charge

\footnotetext{
${ }^{2}$ Thomson-CSF 7852
} 
in one pixel the excess charge falls down the anti-blooming drain rather than spilling into the adjacent pixels (see fig. 2c). Approximately one third of the area of each pixel is taken up by the anti-blooming electrodes and is therefore not photosensitive. This does not cause a significant loss of resolution because the light from a single photoelectron at the input of the first II is usually spread over several pixels. In the conventional operation of CCDs, the image can only be cleared by clocking the charge out of the image zone. This would be too slow for our application, therefore we use the antiblooming facility to make a fast clear. If the anti-blooming voltage $V_{E}$ and the image zone clock voltage $V_{\Phi P}$ are pulsed simultaneously as shown in fig. 4 , then all the charge in the pixels will be forced down the anti-blooming drain as illustrated. To simulate the operation of the detector at the Collider, all the test beam data were taken with a fast clear pulse of $1 \mu \mathrm{s}$ duration every $4 \mu \mathrm{s}$. If the beam-trigger occurred during a period of $2 \mu \mathrm{s}$ around the fast clear pulse then the trigger was vetoed.

After receiving a trigger the following sequence is used to read out the image (see fig. 3). First the image zone charge is transferred to the memory zone. Then the content of the memory zone is transferred line by line to the shift register, which is clocked at a rate of $4 \mathrm{MHz}$. Charge to voltage conversion for each pixel is achieved using a floating diode located at the shift register output. This operation is repeated until the entire image is read out. Thus at the shift register output there is a train of pulses, called the video output, which contains the information of the charges stored in the pixels. The complete sequence takes $\sim 10 \mathrm{~ms}$. To reduce the dead time for the Collider operation it is planned to increase the shift register clock frequency to $7 \mathrm{MHz}$ and thereby reduce the read-out time to $5.6 \mathrm{~ms}$. The principle of the analogue chain for the video output is illustrated schematically in fig. 3 . The raw video signal from the CCD has a large voltage swing even for pixels in the dark. The track and hold amplifier is therefore used to sample the negative video signal during a fraction of the cycle. This leaves only the true signal to be amplified and digitised as is shown in the insert of fig. 3. An analogue subtraction of the black level is made using the analogue switch and pixels 5 and 6 , which are not photo-sensitive. This is achieved by closing the switch during the output of pixels 5 and 6 of each line and then opening the switch. This has the effect of holding the voltage corresponding to the black level on the capacitor $\mathrm{C}_{2}$ (see fig. 3) during the output of the rest of the video line. The amplifiers are AC coupled to avoid problems of $D C$ level drifts. The analogue switch eliminates rate dependent effects that would otherwise be present with AC coupling. A buffer amplifier with a very large input impedance is used so that the RC time constant is much longer than the time to read out one line $(64 \mu \mathrm{s})$. Therefore the voltage drop during the output of a video line is negligible. The video signal is then amplified by a factor of 10 before being digitised by a flash analogue-to-digital converter (FADC) in the digitiser (see section 2.2.3).

\subsubsection{The Digitiser}

The digitiser is a purpose-built FASTBUS module which converts analogue pixel contents into digital values, optionally applies varying degrees of data reduction and stores the results in a memory for read-out. The input to the digitiser is the video-signal from the CCD electronics, together with three control signals: the pixel clock, the line and the frame synchronisation signals (line sync and frame sync). After passing through a receiver amplifier, the analogue signal is digitised by an 8-bit $20 \mathrm{MHz}$ FADC $^{3}$. The exact time of sampling is determined by the pixel clock, generated by the CCD driver electronics. The FADC reference voltage is set so that $1 \mathrm{ADC}$ count corresponds to $1 \mathrm{mV}$ at the CCD output. The line sync and frame sync signals are used to monitor the integrity of the data. At each line sync, the number of pixel clocks received is checked, and at the frame sync, signalling the end of the frame, the number of lines is verified. If either of these numbers differs from standard values, an error flag is set.

The digitiser utilises a pipe-line approach for high speed processing of the digitised signal, and has three modes of operation corresponding to increasing degrees of data reduction. The mode is selected or changed by a FASTBUS instruction.

\footnotetext{
${ }^{3}$ Thomson-TS8328
} 
Mode 1 : reads out every pixel (218 per line, and 145 lines, making 31610 in all). This is essentially a calibration mode. For calibration runs, no processing is applied to the data; they are simply written into a memory and then read out via FASTBUS by the Aleph event builder (see section 2.2.4). These data are normally used to compile a look-up table of mean pedestal values, one for each pixel.

Mode 2 : reads out every pixel that is above a threshold after data processing (see below). For the final detector, this will be used to obtain pixel-to-fibre maps and as a diagnostic mode, with mode 3 being used for data taking. However most of the test beam data were taken using this mode and some were taken in mode 1.

Mode 3 : in this mode, pixels that are above the threshold after data processing are mapped to fibres. Since each fibre subtends of the order of 12 pixels, contributions from each pixel must be added and stored in memory, along with a fibre number. Mode 3 data are the most compact form of data: for each fibre with non-zero content, a "fibre word" is stored. This contains a 12-bit cumulative pulse-height, a 4-bit hit count (the total number of pixels above threshold for each fibre), a 12-bit fibre number and two overflow flags - one for the cumulative pulse-height and one for the hit count. The mode 3 memory is distinct from that used in the other modes. It is therefore possible to record data using mode 3 simultaneously with mode 1 or 2 , and this was done at the test beam to verify the performance of mode 3.

The first stage of processing applied to the data for modes 2 and 3 is to take the 8-bit output of the FADC and subtract from it the pedestal corresponding to that pixel. Since the first 8 pixels on each line of the CCD are masked, one of them can be used to extract a measure of any low frequency noise picked up in the cable. The following procedure is used: from a pedestal run the mean dark level of pixel 8 in each line is recorded in the pedestal look-up table. During data taking, the difference is recorded for pixel 8 between its actual dark level and its pedestal. This difference can then be subtracted from each of the subsequent pixel contents on that line. This black level compensation effectively reduces noise that has a long period compared with the time duration of a line, e.g. a $50 \mathrm{~Hz}$ pick-up (note that DC offsets and thermal drifts are eliminated by AC coupling the analogue signal). The processed data are then compared with a preset threshold and only those pixels that are above threshold are written into the mode 2 or mode 3 memories.

\subsubsection{Operation of the Data Acquisition}

The data acquisition for the fibre detector is based on the FASTBUS standard. For the tests one FASTBUS crate in the counting room contained a digitiser, an Aleph event builder, a segment interconnect (SI) and a CAMAC-FASTBUS interface (CFI). The event builder is master of the acquisition, setting up the digitiser, waiting for an event then reading it out and monitoring it and finally signalling to the VAX online computer when the data are available for writing to tape. The VAX then reads out the appropriate part of the event builder memory via the CFI and the SI.

The Aleph event builder is a general purpose FASTBUS master based on a 68020 processor with floating point hardware [3]. It consists of two FASTBUS cards, one containing the processor, the FASTBUS master interface, two serial ports, an Ethernet interface and $512 \mathrm{~kb}$ of program memory. The second card contains a $512 \mathrm{~kb}$ fast dual-ported memory. One port is connected to the 68020 and the other to a FASTBUS cable segment. A special FORTRAN software package was written to allow communication with the digitiser to be performed easily.

The program to control the digitiser and the data acquisition is completely menu-driven. In the initialisation phase, digitiser parameters (such as mode, threshold etc) are set and checked, and data acquisition and monitoring parameters are defined. The data acquisition can proceed in two ways, with or without event recording on the VAX. For setting up and debugging the VAX is not used, the data are monitored using the Aleph event builder only.

At the beginning of each data-taking period, the CCD pixel pedestals are determined from a run in mode 1 . They are loaded into the appropriate memory of the digitiser and can also be transferred to the VAX, for storage in a disc file for later recovery. 
The pixel-to-fibre map values are determined by offline analysis, as is described in section 3.1. The values are transferred to the VAX and hence to the event builder. The latter then loads the map into the digitiser for use in mode 3 .

For the tests the data taking for particle or fiducial triggers is done using the digitiser in mode 2 or modes 2 and 3 simultaneously. Because of the high stability and low noise of the electronics, a threshold of only $1 \mathrm{ADC}$ count is used. For each event, the status information and the appropriate digitiser memories are read out by the event builder. Monitoring is done on either all or a fraction of the events with various options, e.g. decoded dumps of $C C D$ regions, histograms, two-dimensional event displays. At the end of each run, the histogram buffer can optionally be transferred, through the FASTBUS system, to the online computer and stored for later display.

\subsection{Performance of the Electronics}

The noise on pixels, measured during pedestal runs, is typically $0.35 \mathrm{ADC}$ counts (r.m.s.), which corresponds to $0.35 \mathrm{mV}$ at the output of the $\mathrm{CCD}$, and the measured values are very stable. As will be described in section 3.4, the signal from a single photoelectron produced at the photocathode of the first II corresponds to $\sim 40 \mathrm{ADC}$ counts. The signal of a fibre is spread over typically 12 pixels, so the signal to noise ratio is good even for a single photoelectron. This is illustrated by the raw CCD event displays for muons shown in fig. 5 in which the tracks are clearly visible above the noise. With the short II integration time used, the effects of II noise are negligible. During the test beam running a single fast clear pulse removed $\sim 90 \%$ of the pixel charge. Typically there were many fast clear pulses between particles crossing the detector and thus the efficiency of the fast clear approached $100 \%$. Therefore this small inefficiency did not significantly affect the test data. For operation at the Collider it will be essential to have the fast clear efficiency $\varepsilon_{\mathrm{fc}}$ close to $\sim 100 \%$ after a single fast clear pulse. After the test beam run it was discovered that by raising the peak of the pulse on $V_{E}$ to $22 \mathrm{~V}$ (see fig. 4) one can obtain $\varepsilon_{\mathrm{fc}} \sim 99.5 \%$.

\section{RESULTS}

In this section results from the off-line analysis of the $\mu^{-}, \pi^{-}$and $\mathrm{e}^{-}$beam data are given. The results are complemented with additional information obtained from measurements in which the read-out was illuminated by a single fibre. The following subjects are discussed: the precision of pixel-to-fibre mapping, the position resolution, the response to minimum ionising particles, the efficiency of the tracking part and the performance of the preshower detector.

\subsection{Pixel-to-Fibre Map}

To find the positions of the fibres on the CCD, data from fiducial runs are used. From the accumulation of the total charge in typically a hundred fiducial events the central column $\mathrm{c}_{\mathrm{c}}$ and line $\ell_{\mathrm{c}}$ of each fiducial fibre are found. Within a window of \pm 4 pixels around $c_{c}$ and $\ell_{c}$ a centre-of-gravity calculation yields the position of the fiducial fibre. These positions are compared with their theoretical values in a fitting routine that describes the demagnification and distortion of the image in the image intensifier system. The demagnification of a spot on the picture is a function of its radius on the II entrance window. Several functions were tried to describe this effect. The one that describes the data best is:

with

$$
R_{C C D}=A \cdot R_{i i}\left(1+B \cdot R_{i i}{ }^{3}\right) \text {, }
$$

$$
\begin{array}{ll}
\mathrm{R}_{\mathrm{CCD}}= & \text { the radius on the } \mathrm{CCD}(\mathrm{mm}), \\
\mathrm{R}_{\mathrm{ii}}= & \text { the radius on the II input }(\mathrm{mm}), \\
\mathrm{A}= & 0.0699, \text { the demagnification at } \mathrm{R}_{\mathrm{ii}}=0, \\
\mathrm{~B}= & 2.015 \cdot 10^{-6} \mathrm{~mm}^{-3} \text {, describes the distortion. }
\end{array}
$$


The parameters A and B depend on the focus and zoom voltage settings of the first image intensifier. The above values correspond to a setting of $1.17 \mathrm{kV}$ on the focus and $2.4 \mathrm{kV}$ on the zoom electrode with respect to the cathode. Apart from the demagnification and the distortion, the fitting routine takes care of the absolute position of the coupling plate on the II chain. It allows for a line-offset, a column-offset and a rotation.

The $\chi^{2}$ per degree of freedom is close to 1 if an error of 0.5 pixels on the determination of the fiducial positions is assumed in the fit. Using a higher order polynomial in $\mathrm{R}_{\mathrm{ii}}$ does not give a significant improvement in the quality of the fit. The distribution of residuals has a mean of $0.18 \mathrm{~mm}$ and an r.m.s. of $0.11 \mathrm{~mm}$ (where the residuals are measured at $\mathrm{II}_{1}$ ). Starting from the arrangement of the fibres in the fibre coupling plate, the CCD positions of all fibres are determined using the result of the fit. For the pixel-to-fibre map every pixel is assigned to the nearest fibre, provided the distance from the pixel centre to the fibre centre amounts to less than $75 \mu \mathrm{m}$. Typically 9 to 14 pixels are assigned to a single fibre, inversely proportional to the local surface demagnification of the image intensifier which depends on $R_{\mathrm{ij}}$.

\subsection{Track Fit}

As most of the test data have been taken in digitiser mode 2, a mode 3 data compression is applied offline. A software threshold of 2 ADC counts per pixel is set. The pulse-height information from the first 18 layers is used to find tracks in the fibre stack. Since in the test beam the direction of the particle trajectory is known, the total charge observed in the tracking part of the fibre stack is projected along the beam direction $(z)$ onto a plane coinciding with the first fibre layer. The maximum pulse-height observed in the resulting histogram determines the central fibre $Y_{c}$ of the track. Events with more than one track are rejected, by looking for fibre-bins outside a road of $\pm 2 \mathrm{~mm}$ around $Y_{c}$, which have a charge content larger than $1 / 3$ of the summed pulse-height in $Y_{c}$. In each layer the fibre $F_{m}$ with the maximum pulse-height is found in a road of $\pm 2 \mathrm{~mm}$ around $Y_{c}$. Then a centre-of-gravity calculation is done using pulse-heights in $F_{m}$ and its two neighbours on each side to find a coordinate $Y_{\text {layer }}$ in each layer. Finally the track parameters are found using an unweighted least squares fit to the $Y_{\text {layer }}$ values.

\subsection{Space Resolution}

We have used two methods to study the space resolution of the fibre detector. One method compares the coordinates in each layer with the predicted y-coordinate of the fitted track. For the other method the 18 layers are treated as two separate interleaved sets of 9 layers and the track parameters obtained with each set are compared.

Fig. $6 \mathrm{a}$ shows a histogram of the comparison between the positions $Y_{\text {layer }}$ in the first 18 layers and the $y$-position $Y_{\text {track }}$ of the fitted track. For this figure data are used in which the fibre stack is rotated by 14 degrees around the fibre axis to avoid effects introduced by the alignment of the fibres in the different layers with respect to the beam incidence. All 18 layers are used in the fit. The FWHM of the distribution is $0.86 \mathrm{~mm}$, representing the space resolution per layer. As the data are not fully described by a single Gaussian distribution, we have fitted the histogram to a sum of three Gaussians: a central one with normalisation $n_{1}$ and r.m.s. $\sigma_{1}$ plus two identical satellites at $\pm 1 \mathrm{~mm}$ offset with normalisation $\mathrm{n}_{2}$ and r.m.s. $\sigma_{2}$. The curve in fig. 6 a represents the result of the fit, giving $\mathrm{n}_{2} / \mathrm{n}_{1}=0.028$, $\sigma_{1}=0.35 \mathrm{~mm}$ and $\sigma_{2}=0.58 \mathrm{~mm}$. This indicates that in $6 \%$ of the cases the centre-of-gravity is not assigned to the correct fibre due to the resolution of the read-out chain. As the divergence of the beam is negligible, the spread in the fitted track angles measures the angular precision of the detector. This is shown in fig. $6 \mathrm{~b}$ together with the result of the fit to a Gaussian distribution giving $\sigma=13 \mathrm{mrad}$. For the second method the distribution of the fitted track angles for each of the two sets of 9 layers gives $\sigma=16 \mathrm{mrad}$, whereas for the distribution of the difference between the two angles $\sigma=20 \mathrm{mrad}$. If the errors were purely statistical, the three values of the angular resolution would increase by factors of $\sqrt{ } 2$. Fig. $6 c$ shows the distribution of the difference $Y_{\text {track1 }}-Y_{\text {track2 }}$ between the postions of the two 
9-layer fitted tracks in the medium plane of the fibre stack. The distribution is well described by a Gaussian with $\sigma=0.20 \mathrm{~mm}$. Thus we conclude that the track position resolution in the fibre detector is better than $0.20 \mathrm{~mm}$.

\subsection{Single Fibre Measurements}

As an additional check of the resolution of the read-out system, separate measurements have been done by illuminating the entrance window of the first image intensifier with a single $1 \mathrm{~mm}$ diameter optical fibre. The fibre was placed at 13 different positions on the entrance window to check the uniformity of the result. The position resolution of $0.39 \mathrm{~mm}$ varies by about $10 \%$ over the surface of the read-out. It is found that $79 \pm 2 \%$ of the light output from the single fibre is detected in the pixels assigned to it in the pixel-to-fibre map. This value drops to $\sim 70 \%$ in the space on the CCD corresponding to the $1 \mathrm{~mm}$ diameter fibre itself. From the amount of light which is detected outside the space assigned to the hit fibre in the map, on average $70 \%$ is assigned to neighbouring fibres within the same fibre layer.

The single fibre setup has also been used to measure the response of the read-out system to a single photoelectron at the output of the first photocathode. The light from the flasher used to illuminate the fibre is attenuated until only $\sim 10 \%$ of the flashes produced any light on the CCD. Events with non-zero charge on the CCD are used to measure the response of the system to single photoelectrons. The charge is integrated in a region of 20 pixels about the fibre centre. A background subtraction is made using a control region far from the fibre. The result is shown in fig. 7a. The mean charge is $43 \mathrm{ADC}$ counts with an estimated systematic error of 3 and the r.m.s. is 30 counts. The distribution has not been corrected for events with two or more photoelectrons. If one assumes that the distribution of the number of photoelectrons obeys Poisson statistics the mean charge per photoelectron is $40 \pm 3$ ADC counts.

\subsection{Fibre Charge and Detection Efficiency}

The muon beam data are used to measure the response of the SFD to minimum ionising particles (MIP). The charge distribution per hit fibre and the detection efficiency are determined. Data taken at different impact angles $\theta$ (see fig. 1) are used to measure the attenuation length of the fibres. For each event cuts are applied on the beam counters and chambers to reject triggers with two particles. A track fit is made as described in section 3.2 and is used to predict the hit fibres in each layer. To allow for the effects of finite II resolution the charge in a layer is defined as the sum of the charges in the predicted hit fibre plus the two neighbouring fibres on each side. A control region of the same size far from the track is used to measure background charge.

We will first describe the results of the data taken at $\theta=90^{\circ}$. The charge distribution for single fibre layers in the tracking detector is shown in fig. $7 \mathrm{~b}$. The distribution shows a spike at zero charge which corresponds to zero photoelectrons from the first photocathode. Although on average the signal is very much larger than the noise, the distribution is so broad that it is important to use a low ADC threshold to avoid extra inefficiency. The shape of the distribution at low charge is dominated by the single photoelectron response. The average charge is 112 ADC counts, which is 2.8 times the average charge for a single photoelectron (see fig. 7a and section 3.4).

Fig. 7c shows the detection efficiency for all 24 layers of the detector. There is no significant variation over a layer. The efficiency is lower in the preshower, where the II gain is reduced by the light attenuator. This is mainly due to the effect of the ADC threshold cut. The primary purpose of the preshower part is to identify electrons by detecting their early shower development. So the small loss of efficiency in this region for particles which do not shower is not very important.

In the tracking part the efficiency is dominated by Poisson statistics in the number of photoelectrons after the first photocathode. Therefore the measured efficiency can be used to calculate the aver- 
age number of photoelectrons $\mathrm{dN} / \mathrm{dt}$ generated per unit thickness of fibre traversed $t$. It is assumed that $\mathrm{dN} / \mathrm{dt}$ does not depend on the position within a fibre. Thus the measured inefficiency is given by a convolution of a Poisson distribution with the length traversed by the track in the fibre. Under those assumptions the measured efficiency corresponds to an average of $\sim 2.8$ photoelectrons produced in the photocathode of the first II per scintillating fibre traversed (or 3.6 per $\mathrm{mm}$ ) at a distance of $1.3 \mathrm{~m}$ from the II input. This is in excellent agreernent with the charge measurement described above.

Although the charge distribution in a layer is rather broad, the charge per track (averaged over the 18 tracking layers) shows a much narrower distribution (see fig. 8). Also shown in fig. 8 is a prediction for 2 MIPs using a convolution of the 1 MIP distribution with itself. This illustrates that the detector could also be used to help in the separation of single electrons from photon conversions occurring before the SFD.

The fibre efficiencies and the CCD output average charge for different sets of $(\theta, \ell)$ values (see fig. 1) are listed in table 2. The efficiency has a minimum around $\theta=90^{\circ}$. The efficiency is larger for $\theta>90^{\circ}$ than for $\theta<90^{\circ}$ because of the smaller distance $\ell$ to the read-out.

The data at different values of $\theta$ and $\ell$ are also used to measure the attenuation length of the fibres. The average charge is corrected by a factor $\sin \theta$ to allow for the different thickness of fibre traversed. The results are shown in fig. 9. A reliable determination of the attenuation length is not possible because of a lack of knowledge of the reflectivity $\varepsilon_{\mathrm{r}}$ of the reflector at the end of the fibres opposite the read-out. The fitted value of the attenuation length, $\lambda_{\text {att }}$, varies from $1.7 \mathrm{~m}$ to $1.4 \mathrm{~m}$ for $0.2<\varepsilon_{\mathrm{r}}<0.5$.

\begin{tabular}{|cccc|}
\hline \multicolumn{3}{c|}{ Table 2: Fibre data for different sets of $(\theta, \ell)$} \\
$\theta$ & $\ell$ & $\begin{array}{c}\text { Efficiency } \\
(\%)\end{array}$ & $\begin{array}{c}<\text { Charge }> \\
\text { (ADC counts) }\end{array}$ \\
& $(\mathrm{mm})$ & & \\
$28^{\circ}$ & 2080 & 96.2 & 186 \\
$45^{\circ}$ & 1710 & 93.4 & 143 \\
$90^{\circ}$ & 1300 & 90.9 & 112 \\
$135^{\circ}$ & 910 & 97.4 & 220 \\
$152^{\circ}$ & 520 & 98.7 & 440 \\
& & & \\
\hline
\end{tabular}

\subsection{Performance of the Preshower Detector}

The purpose of the preshower part of the detector is to recognize the presence of an electromagnetic shower and give a good determination of the starting point of electromagnetic showers. Requiring a good match between the incident track and the preshower cluster significantly reduces the probability of mistaking a charged hadron with a nearby photon for an electron. Three single event displays for electrons are shown in fig. 10.

In order to reconstruct clusters the charge in the preshower region is projected onto a plane coinciding with the first fibre layer. Several cluster algorithms were tested in order to find showers independently of the information from the tracking part. The simple algorithm used is to connect all adjacent fibre bins with a preshower charge above a certain cutoff, $\mathrm{Q}_{1}$, provided at least one bin is above a second threshold, $\mathrm{Q}_{2}$. The value of $\mathrm{Q}_{2}$ is chosen low enough (about $15 \%$ of a typical MIP pulse-height) to maintain a high efficiency $(\sim 95 \%)$ for minimum ionising tracks, while $Q_{1}$ is $0.3 \times Q_{2}$. 
The distribution of the shower charge for $40 \mathrm{GeV}$ electrons is shown in fig. 1la (note the logarithmic abscissa). It has been normalised to the average shower charge for $160 \mathrm{GeV}$ muons (1 MIP). The corresponding distribution for $40 \mathrm{GeV}$ pions is also shown in fig. 1la. Fig $11 \mathrm{~b}$ shows a comparison between the shower charges for $10 \mathrm{GeV}$ and $40 \mathrm{GeV}$ electrons. The detection efficiency for electrons versus the pion efficiency is shown in fig 12 for various values of the minimum cluster charge. The preshower detector can provide a large rejection factor against single pions, which is however strongly correlated with the calorimeter rejection in the UA2 detector.

For $40 \mathrm{GeV}$ incident electrons the centre of gravity of the clusters, $Y_{\text {centroid, is compared with }}$ the extrapolated position of the track, $Y_{\text {track }}$, found before the converter. If several showers are found the one with the highest pulse-height is chosen. The distribution of the distance between the track and the cluster-centroid ( $Y_{\text {track }}-Y_{\text {centroid }}$ ) is shown in fig. 13a together with a fitted Gaussian. The width of this distribution, $\sigma=0.76 \mathrm{~mm}$, includes the error in the fitted track and is much smaller than the average shower profile shown in fig. $13 \mathrm{~b}$ together with the average cluster profile of $160 \mathrm{GeV} \mu^{-}$.

The large width of the electromagnetic shower profiles makes it very difficult to separate a nearby hadron from a photon using the preshower information alone. Consider, for example, a MIP track at 2 $\mathrm{mm}$ distance from a shower. It will cause a shift of about $0.1 \mathrm{~mm}$ of the cluster-centroid position. However, it will be possible to separate such a track from the photon shower at the level of two to three standard deviations using the mismatch between the fitted track and the charge cluster $(\sigma=0.76$ $\mathrm{mm})$.

\section{CONCLUSIONS}

A prototype of the scintillating fibre detector to be used in the UA2 upgrade has been tested in beams of muons, pions and electrons. The position resolution in the tracking part of the detector is $0.35 \mathrm{~mm}$ for a single fibre layer and better than $0.20 \mathrm{~mm}$ for tracks. The angular resolution is $13 \mathrm{mrad}$. The efficiency of a single fibre to detect a minimum ionising particle varies from $91 \%$ for normal beam incidence at $1.3 \mathrm{~m}$ distance from the read-out to $99 \%$ for $152^{\circ}$ incidence close to the read-out. The summed pulse-height for a track observed in the tracking part has a narrow distribution and can be used to identify photon conversions. The preshower detector gives a response 20 times higher for 40 $\mathrm{GeV}$ electrons than for pions, which can be used to obtain a high rejection factor against pions while retaining a good detection efficiency for electrons. The match between the centroid of an electron shower in the preshower detector and the track observed in the tracking part has a resolution of 0.76 $\mathrm{mm}$ (r.m.s.) and will be used to distinguish between an electron and a charged hadron with a nearby photon.

\section{Acknowledgments}

It is a pleasure to thank our UA2 colleagues for their constant support and understanding. We thank the technical staff of CEN Saclay for the production of the fibres. We wish to thank J.C. Berset for his invaluable help in the early stages of this work. 


\section{Appendix}

\section{MCP image intensifier properties}

The second image intensifier $\left(\mathrm{II}_{2}\right)$ within each chain provides the bulk of the light amplification as it contains a micro channel plate (see section 2.2.1), which gives a large electron multiplication. A schematic drawing of $\mathrm{II}_{2}$ is shown in fig 14 . The relevant limitations because of the high gain of $\mathrm{II}_{2}$ are:

\section{Saturation}

For large photoelectric currents, the voltage across the MCP cannot be maintained due to the plate resistivity, thus the MCP gain is decreased.

Lifetime (or life-charge)

The MCP and the photocathode both deteriorate due to effects induced by the photoelectric current amplified by the MCP, thus causing a loss of gain

$$
\mathrm{G}=\mathrm{G}_{0} \cdot \mathrm{e}^{-\mathrm{q} / \mathrm{q}_{0}}
$$

where $\mathrm{q}$ is the charge density integrated over time at the MCP output and $\mathrm{q}_{0}$ is an empirical parameter. The deterioration of the photocathode is not sharply localized at the light impact point.

The saturation and lifetime properties of $\mathrm{II}_{2}$ were studied in a separate experiment and the results were compared to the conditions expected during the Collider operation. A uniform light source (diameter $17 \mathrm{~mm}$ ) of variable intensity was made of an electroluminescent (EL) plate, a filter $(\lambda=$ $450 \pm 25 \mathrm{~nm})$ and a fibre optic disc. A photomultiplier was used to calibrate the relative light yield of the source as a function of the voltage applied to the EL plate. The light source was then put in contact with the fibre optic-input window of $\mathrm{II}_{2}$ and the following three quantities accessible to measurements were used during the study:

1. the photoelectric current produced in the photocathode, $i_{\mathrm{pc}}$,

2. the electric current after MCP amplification, i $\mathrm{MCP}$,

3. the photon gain of the II, $\mathrm{G}=$ (\# photons out / \# photons in).

The latter quantity was measured by comparing the response of the PM to the light source viewed directly and with amplification by $\mathrm{II}_{2}$. A filter of calibrated attenuation for the phosphor light spectrum was inserted between the II and the PM for the amplified measurement to avoid PM saturation.

The variation of the photon gain as a function of the light source intensity was measured. The results are shown in fig. 15 as a function of the input photon flux ${ }^{1}$, the relevant parameter for a comparison with the Collider operation conditions. The gain, which decreases only by $20 \%$ for an increase of the flux from $5 \times 10^{6}$ to $5 \times 10^{9}$ photons $\mathrm{cm}^{-2} \mathrm{~s}^{-1}$, falls sharply for larger fluxes. For the Collider operation we plan to work with a total photon gain of 40000 in the II chain, which corresponds to a gain of 800 for $\mathrm{II}_{2}$, instead of $\sim 1200$ used for the test. The expected gain variation for the Collider operation is given by the dashed curve in the figure.

In order to evaluate the input photon flux onto $\mathrm{II}_{2}$ during the Collider operation the following assumptions were used: inelastic $\bar{p}$ p cross section $=41 \mathrm{mb}$; fraction of fibres hit in the tracking region $=2 \%$ per interaction; photon multiplicity per fibre hit averaged over the incidence angle $=50$; effective photon gain of $\mathrm{II}_{1}=8$. The fraction of fibres hit includes an allowance of about a factor 2 for spurious hits. With those assumptions and for an expected maximum luminosity $\mathscr{L}=4 \times 10^{30}$

1 In order to evaluate the input photon flux the following correspondence was used:

$l e^{-}$out $M C P \rightarrow n$ photons out $\rightarrow n / G$ photons in,

where $n$ is the number of photons produced by a $6 \mathrm{kV}$ electron in the phosphor of $\mathrm{II}_{2}$ times the transmission of the fibre optic output window. A value of $n=15$ was used. The uncertainty on $n$ was estimated to be $\pm 20 \%$. 
$\mathrm{cm}^{-2} \mathrm{~s}^{-1}$, the expected fluxes at the input of $\mathrm{II}_{2}$ are:

$$
\begin{array}{ll}
\text { SFD tracking } & \sim 2 \times 10^{9} \text { photons } \cdot \mathrm{cm}^{-2} \cdot \mathrm{s}^{-1} \\
\text { SFD preshower } & \sim 6 \times 10^{9} \text { photons } \mathrm{cm}^{-2} \mathrm{~s}^{-1} .
\end{array}
$$

In addition the light of the preshower part will be attenuated by a factor 4 in front of $\mathrm{II}_{2}$. In conclusion, the saturation does not seem to be a problem for the Collider operation.

The same set-up was used to study the ageing properties of $\mathrm{II}_{2}$ for large integrated light exposure. In order to be sensitive to possible smearing effects, a fraction of the $18 \mathrm{~mm}$ diameter II input window was masked by a strip $5.6 \mathrm{~mm}$ in width. The intensity of the light source was adjusted to about $3 \times 10^{9}$ photons $\mathrm{cm}^{-2} \mathrm{~s}^{-1}$. The II light output was constantly monitored with a PM, whose output signal was recorded. The gain and the anode current of $\mathrm{II}_{2}$ were checked periodically under standard conditions $(\mathrm{MCP}$ voltage $770 \mathrm{~V})$.

The ageing test was divided into three periods. During the first and third period the MCP voltage was set only at the start of the period to obtain a gain of about 1200 , whereas for the second period the MCP voltage was reset every day in order to restore the gain to its initial value. Similar adjustments, although less frequent, will be made during the Collider operation. The information about the three periods, as well as the $\mathrm{q}_{0}$ values (see $\mathrm{Al}$ ), which were obtained, are summarized in table 3 . The three measurements of $\mathrm{q}_{0}$ obtained under different conditions are in excellent agreement. We will use as a life charge the average value:

$$
\mathrm{q}_{0}=0.055 \mathrm{C} \cdot \mathrm{cm}^{-2}
$$

For the Collider operation, with an $\mathrm{II}_{2}$ photon gain of 800 , an integrated charge density equal to $\mathrm{q}_{0}$ will correspond to $6.5 \times 10^{15}$ photons $\mathrm{cm}^{-2}$ at the $\mathrm{II}_{2}$ input, and, by using $\mathrm{A} 2$, to an integrated luminosity:

$$
L=13 \mathrm{pb}^{-1}
$$

The complete gain evolution equation for $\mathrm{II}_{2}$ is:

$$
\mathrm{G}=\mathrm{G}_{0} \cdot \mathrm{e}^{\Delta \mathrm{V} / 90} \cdot \mathrm{e}^{-\Delta \mathrm{q} / \mathrm{q}_{0}}
$$

and the available $\Delta \mathrm{V}_{\max } \simeq 300$ Volts for a typical $\mathrm{II}_{2}$ may be used to compensate a loss due to $\Delta \mathrm{q} \simeq$ $3 \mathrm{q}_{0}$, corresponding to an integrated luminosity:

$$
\mathrm{L} \simeq 40 \mathrm{pb}^{-1}
$$

The localisation of the ageing effects was studied by scanning a $\sim 1 \mathrm{~mm}$ diameter light spot across the II photocathode before and after ageing. The variation of the II gain as a function of the light spot position before ageing and after ageing with the mask removed is shown in fig. 16. The profile observed before ageing with the mask on is perfectly consistent with the mask width of $5.6 \mathrm{~mm}$ and a 1.1 $\mathrm{mm}$ diameter light spot. In fig. 16 the dashed curve is the expected profile for an exact localization of the ageing effect. Losses of $20 \%$ to $10 \%$ are seen to extend about $1 \mathrm{~mm}$ inside the masked region. This effect may be due to destructive diffusion within the photocathode material.

The sharing of the ageing damage between the photocathode and the MCP was also studied by measuring after ageing both the photoelectric current $\dot{i}_{\mathrm{pc}}$ and the electric current after MCP amplification $i_{\mathrm{MCP}}$, with mask and with mask removed. From the four measurements is was concluded that the damage was shared roughly equally $(50 \% \pm 10 \%)$ between the photocathode and the MCP. It should be noted that this type of damage to the photocathode is specific to $\mathrm{II}_{2}$. It is closely related to the very small distance between the MCP and the photocathode in $\mathrm{II}_{2}$. 
Table 3: Summary of the lifetime tests

\begin{tabular}{lllcc} 
period & $\begin{array}{c}\text { duration } \\
(\mathrm{hrs})\end{array}$ & \multicolumn{1}{c}{$\begin{array}{c}\mathrm{V}_{\mathrm{MCP}} \\
(\mathrm{V})\end{array}$} & $\begin{array}{c}<\mathrm{i}_{\mathrm{MCP}}> \\
(\mathrm{nA})\end{array}$ & $\begin{array}{c}\mathrm{q}_{0} \\
\left(\mathrm{C} \cdot \mathrm{cm}^{-2}\right)\end{array}$ \\
1 & 140.5 & 770 & 66 & 0.054 \\
2 & 217.5 & adjusted & 70 & 0.052 \\
3 & 260 & 890 & 66 & 0.058
\end{tabular}




\section{REFERENCES}

1. The UA2 experiment at ACOL, The UA2 collaboration, presented by C.N. Booth at the 6th Int. Conf. on $\bar{p}$ p Physics, Aachen, June 30 - July 4 ,1986; to be published by World Scientific Publishing Co., Singapore.

Proposal to improve the performance of the UA2 detector, CERN/SPSC 84-30 (1984).

Proposal to improve the performance of the UA2 central detector, CERN/SPSC 84-95 (1984).

2. Design study of an antiproton collector (ACOL), ed. E.J.N. Wilson, CERN 83-10 (1983).

E. Jones et al., IEEE Trans. Nucl. Sci., Vol NS-30 nr 4 (1983).

3. R. Benetta et al., Proceedings of the FASTBUS Software Workshop, September 23-24, 1985, CERN 85-15, 131, (1985).

4. Optical characteristics of cathode ray tube screens, Electronic Industries Association, 2001 Eye Street N.W., Washington DC 20006, USA. TEPAC publication No. 116A (1985).

\section{FIGURE CAPTIONS}

1. The test setup showing the fibre bundle and the read-out chain. The different angles and positions of beam-incidence are indicated. The enlarged view shows the image intensifier system.

2. Cross-sections through CCD pixels.

3. CCD read-out scheme. The insert illustrates the operation of the track and hold amplifier:

a. Part of the video output from the CCD. Pixels $N$ and $N+1$ are in the dark and pixels $\mathrm{N}+2$ and $\mathrm{N}+3$ are illuminated.

b. The corresponding output of the track and hold amplifier.

4. Fast clear operation:

a. Pulses used.

b. Charge flow.

5. Raw CCD event displays for muon tracks. The circles represent the calculated positions of the fibres on the CCD image. The light level is proportional to the charge content in each pixel.

6.

a. Deviation of the positions measured in the first 18 layers from the coordinate $Y_{\text {track }}$ of the fitted track. The curve shows the result of the fit to three Gaussians as described in the text.

b. Distribution of the fitted track angle measured in the fibre detector. The curve shows the result of the fit to a Gaussian distribution giving $\sigma=13 \mathrm{mrad}$.

c. Difference between the track-positions $Y_{\text {track } 1 \text { and }} Y_{\text {track } 2}$ from track fits using two separate interleaved sets of 9 layers. The curve shows the result of the fit to a Gaussian distribution giving $\sigma=0.20 \mathrm{~mm}$.

7. The distribution of the average charge per track in the tracking part. The dashed curve is the prediction for 2 MIP using a convolution of the measured 1 MIP distribution with itself.

8. a. The charge distribution from single photoelectron events.

b. The distribution of the charge per layer.

c. Mean detection efficiency for minimum ionising particles per layer versus layer number.

9. Corrected average fibre pulse-height $\mathrm{q}_{\mathrm{MIP}} \times \sin \theta$ versus distance $\ell$. The superimposed curve is an exponential fit to the attenuation length with $\varepsilon_{\mathrm{r}}=0.2$ which gives $\lambda_{\text {att }}=1.6 \pm 0.1 \mathrm{~m}$. 
10. Single event displays for electrons. The bin-contents are proportional to the pulse-heights in the fibres. The pulse-height in the preshower region has been multiplied by a factor of 4 to correct for the effect of the attenuator.

11. a. Charge distribution of $40 \mathrm{GeV}$ pions and showers caused by $40 \mathrm{GeV}$ electrons in the preshower detector.

b. Charge distribution of showers caused by 10 and $40 \mathrm{GeV}$ electrons in the preshower detector.

12. Detection efficiencies of $40 \mathrm{GeV}$ electrons and of $40 \mathrm{GeV}$ pions for different minimum cluster charge requirements, shown in the top horizontal scale (MIP), in the preshower.

13. a. Distance between the cluster-centroid and the extrapolated track for electrons.

b. Average profiles and relative sizes of clusters caused by $40 \mathrm{GeV}$ electrons and muons in the preshower part. The charge content of the muon clusters has been scaled by a factor 10 .

14. Schematic drawing of $\mathrm{II}_{2}$ (not to scale) showing the principle of operation.

15. The $\mathrm{II}_{2}$ photon gain as a function of the input photon flux The full curve is an eyeball fit to the data and corresponds to an average gain of 1200 . It has been scaled by a factor of $1 / 1.5$ for the gain and 1.5 for the flux to obtain the dashed curve (average gain 800).

16. The $\mathrm{II}_{2}$ photon gain as a function of the light spot position across the photocathode:- before ageing without the mask; - before ageing with the mask on; $X$ after ageing with the mask removed. The dashed curve is the expected gain profile for a perfect localization of the ageing effect. 

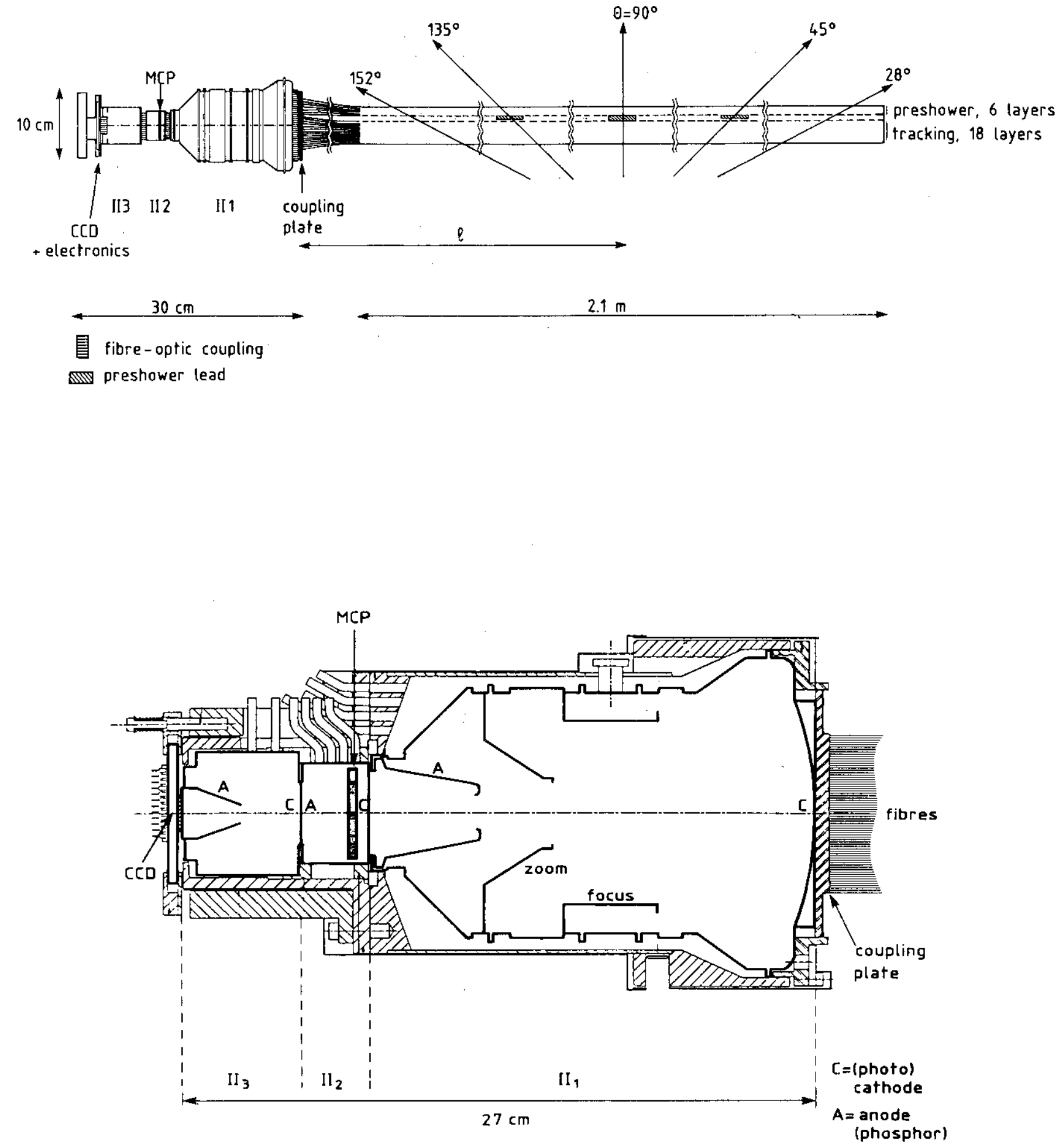

fig. 1 


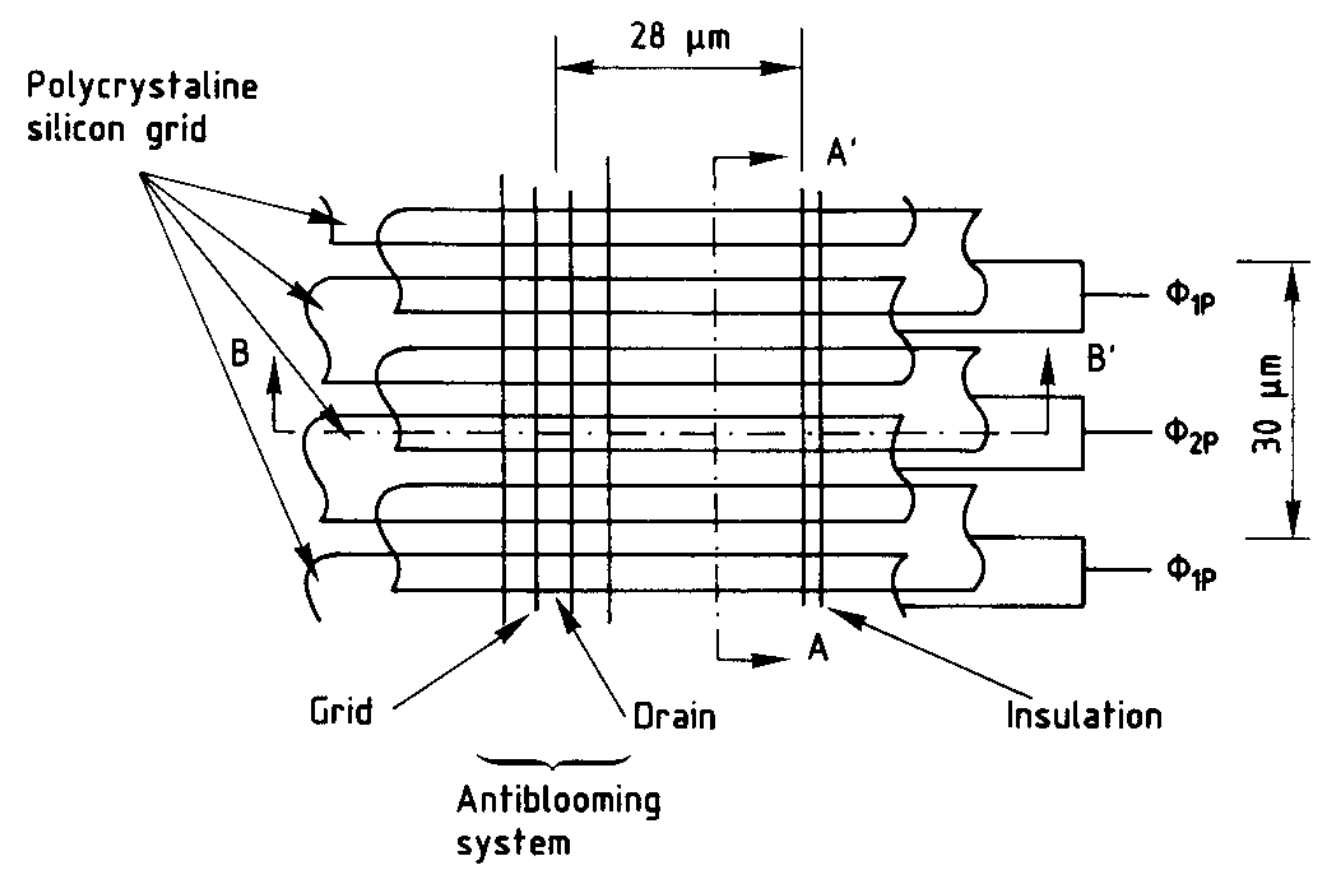

a) Plan view of photoelements (pixels).

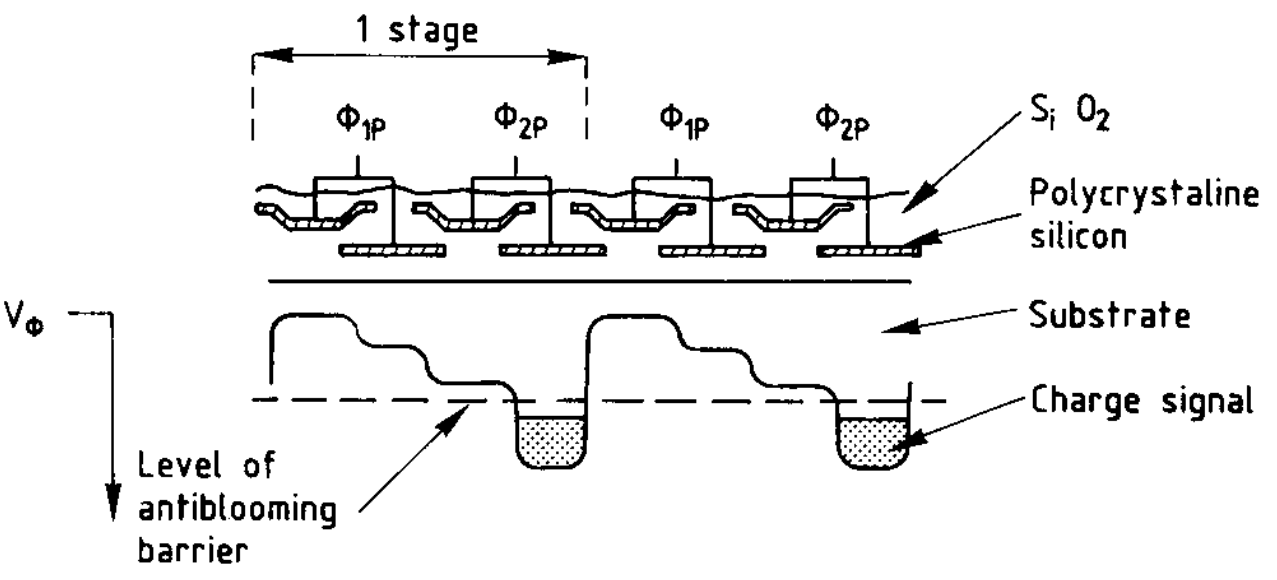

b) Cut $A A^{\prime}$ through pixel and voltage profile.

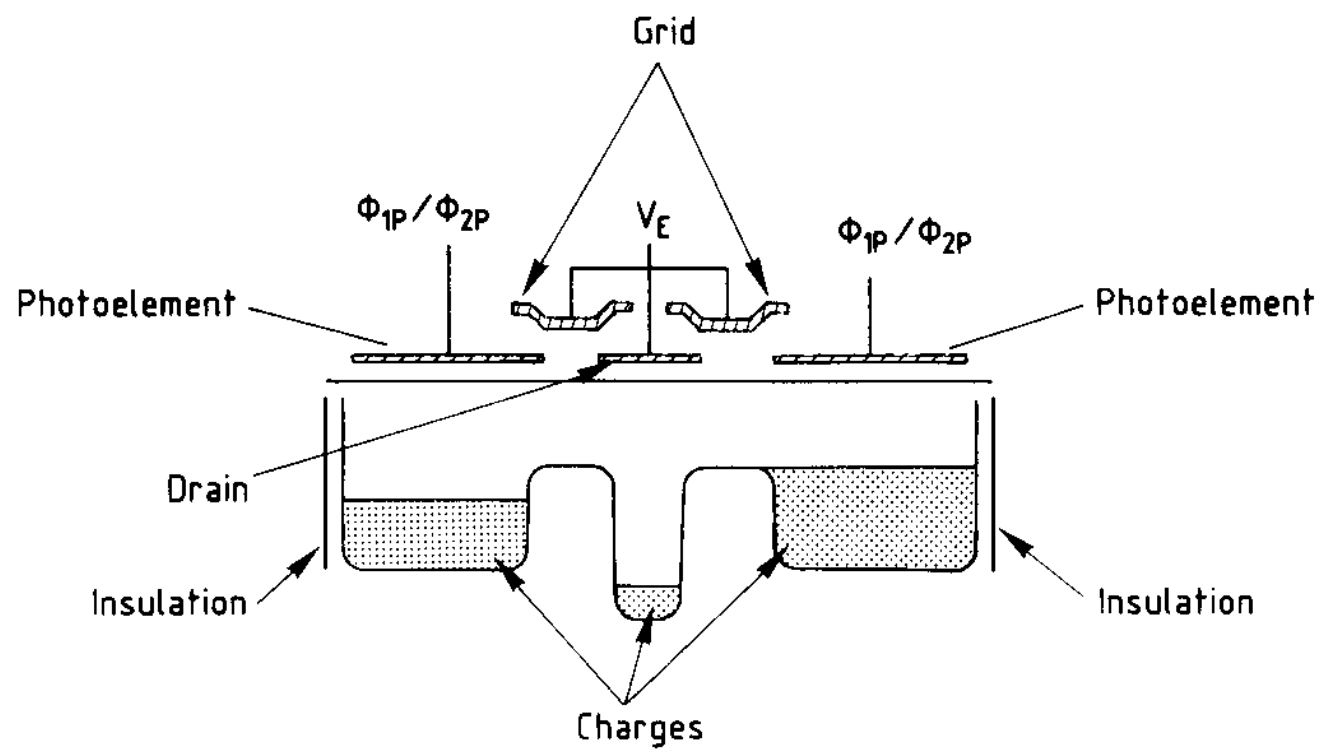

c) Cut BB' through pixel and voltage profiles illustrating antiblooming orain. 


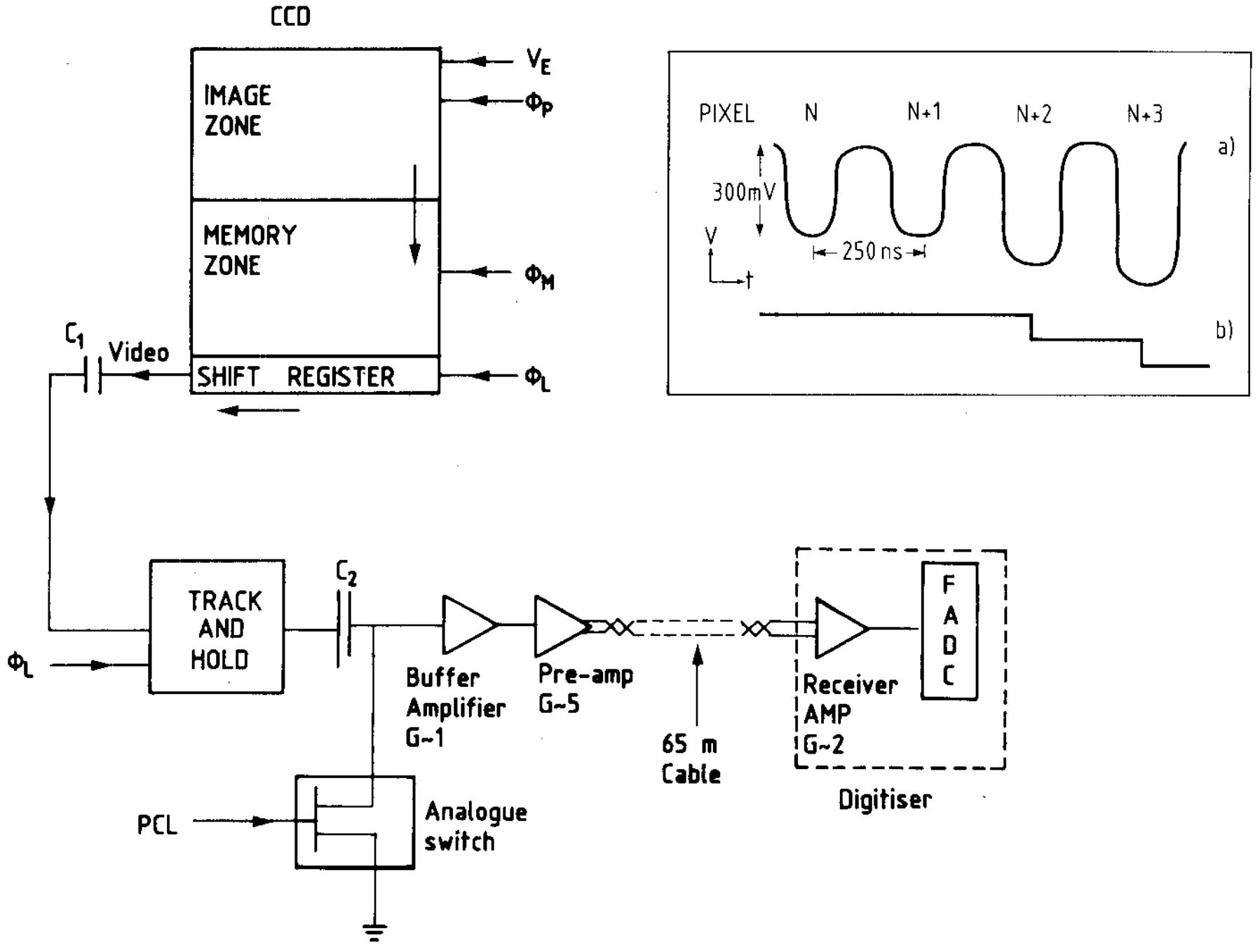

$\begin{array}{ll}V_{E} & \text { anti-blooming Voltage } \\ \Phi_{P} & \text { image zone clock } \\ \Phi_{M} & \text { memory zone clock } \\ \Phi_{L} & \text { shift register clock } \\ V i d e o & \text { video-output from CCD } \\ P C L & \text { pre-clamp signal }\end{array}$


a) Fast clear pulses

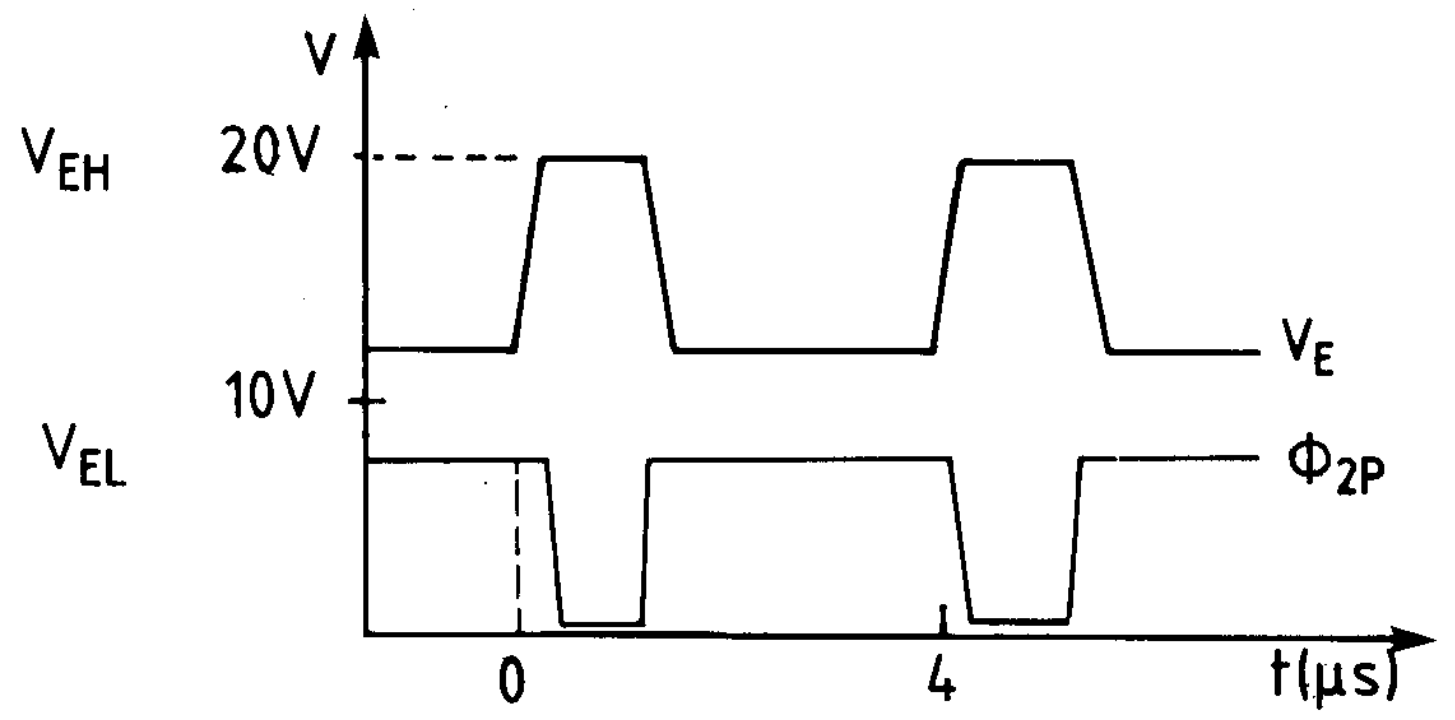

b) Profile of antiblooming barrier for different voltages $V_{E}$

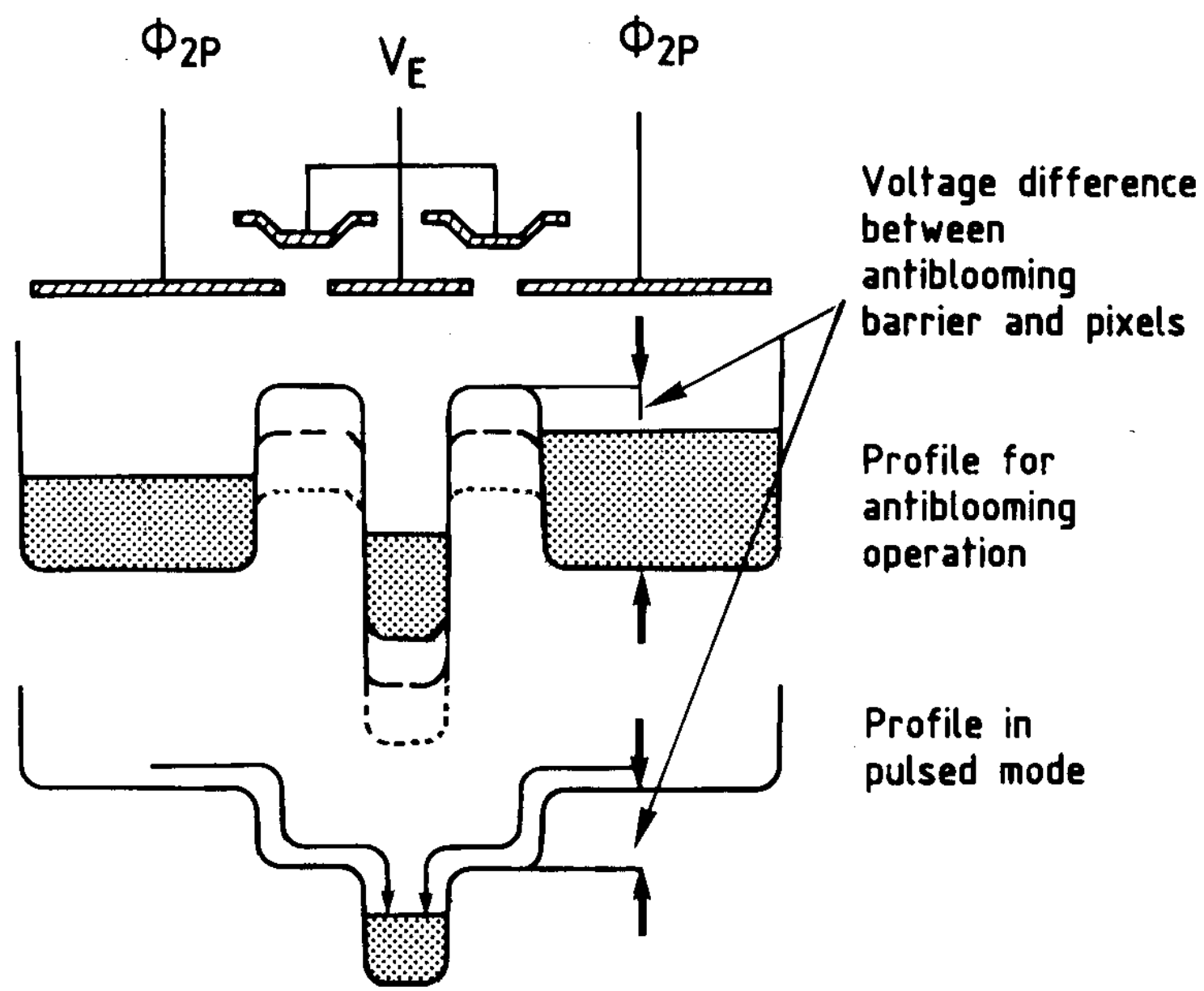




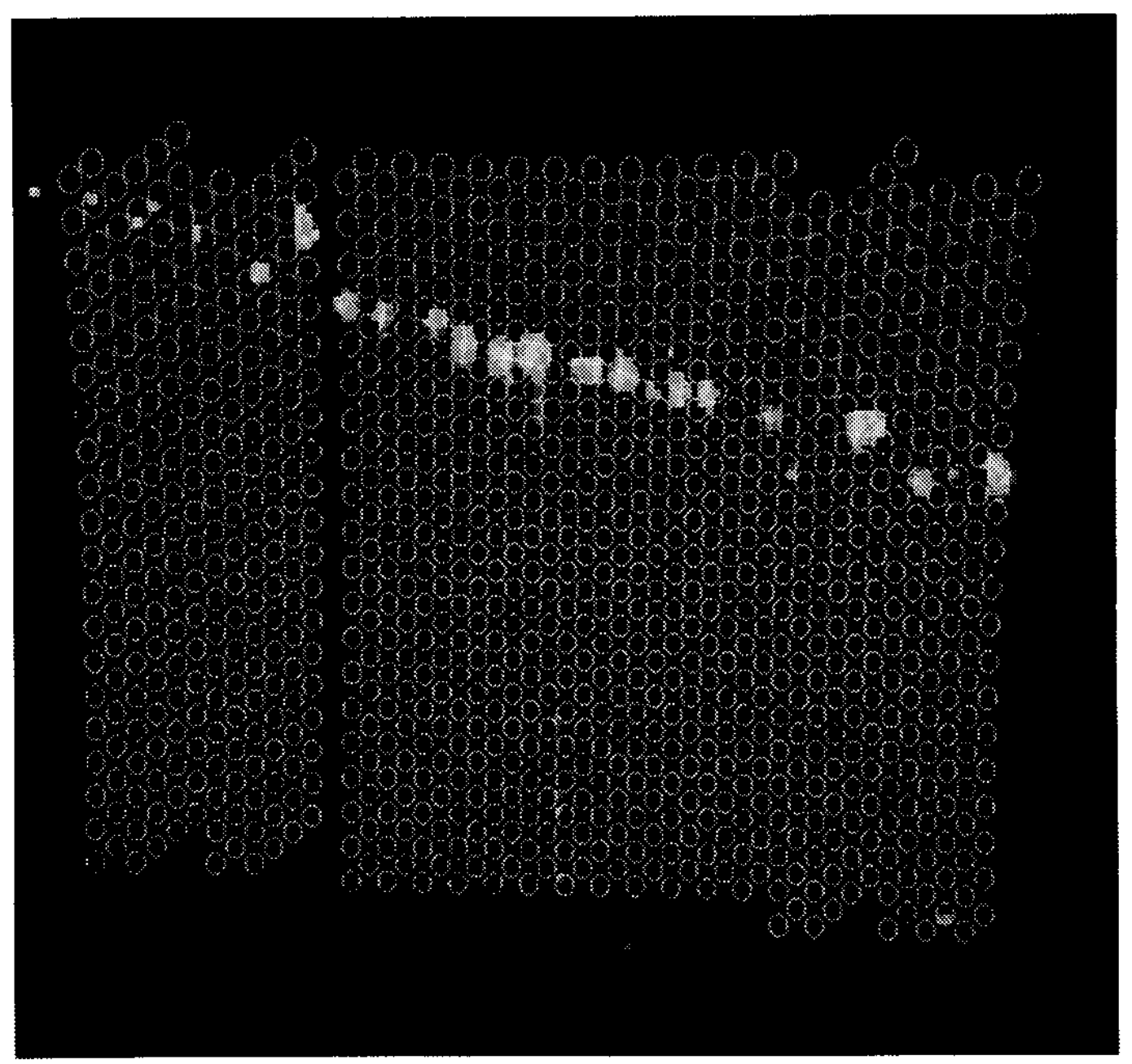

เ

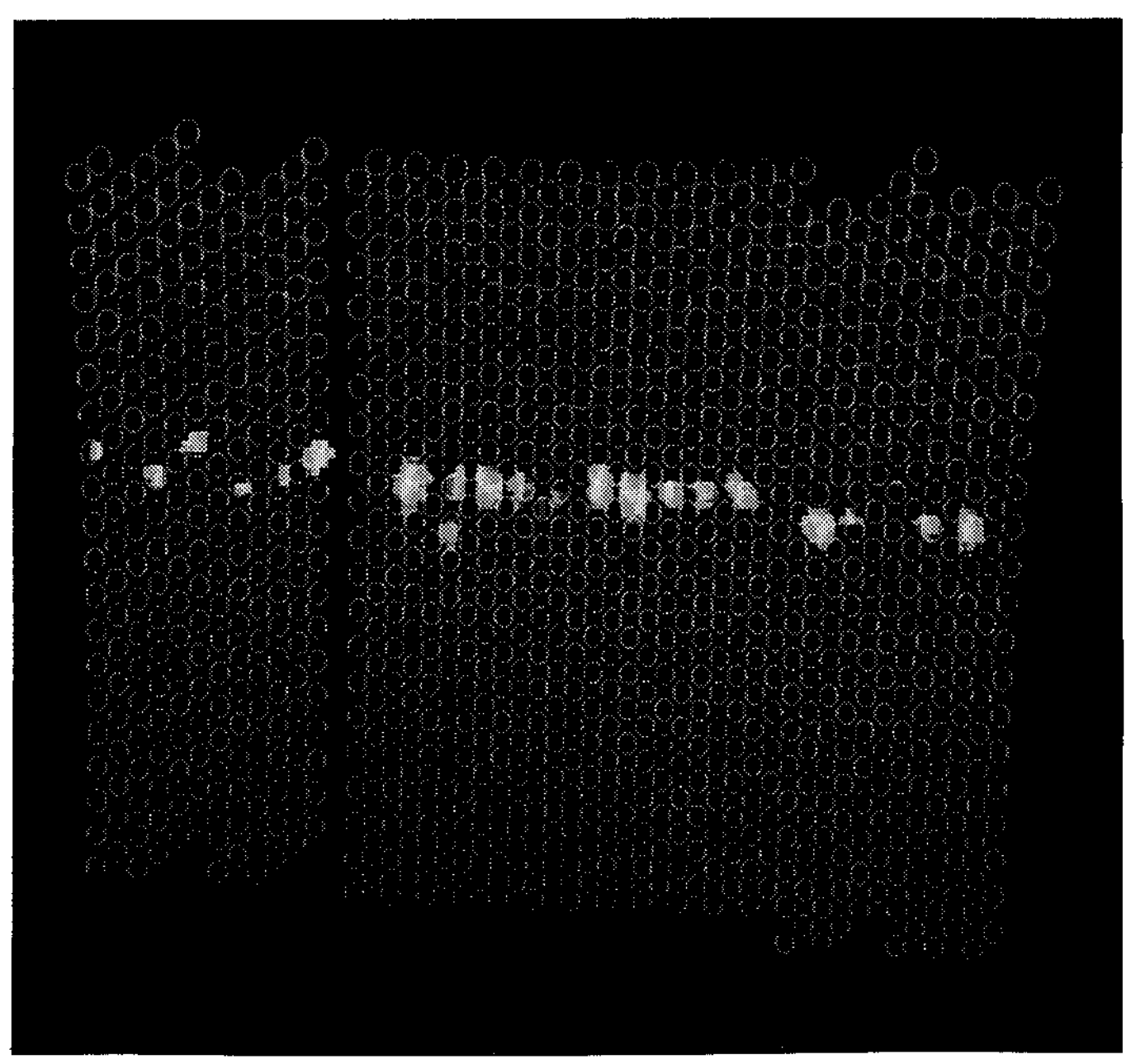

它 

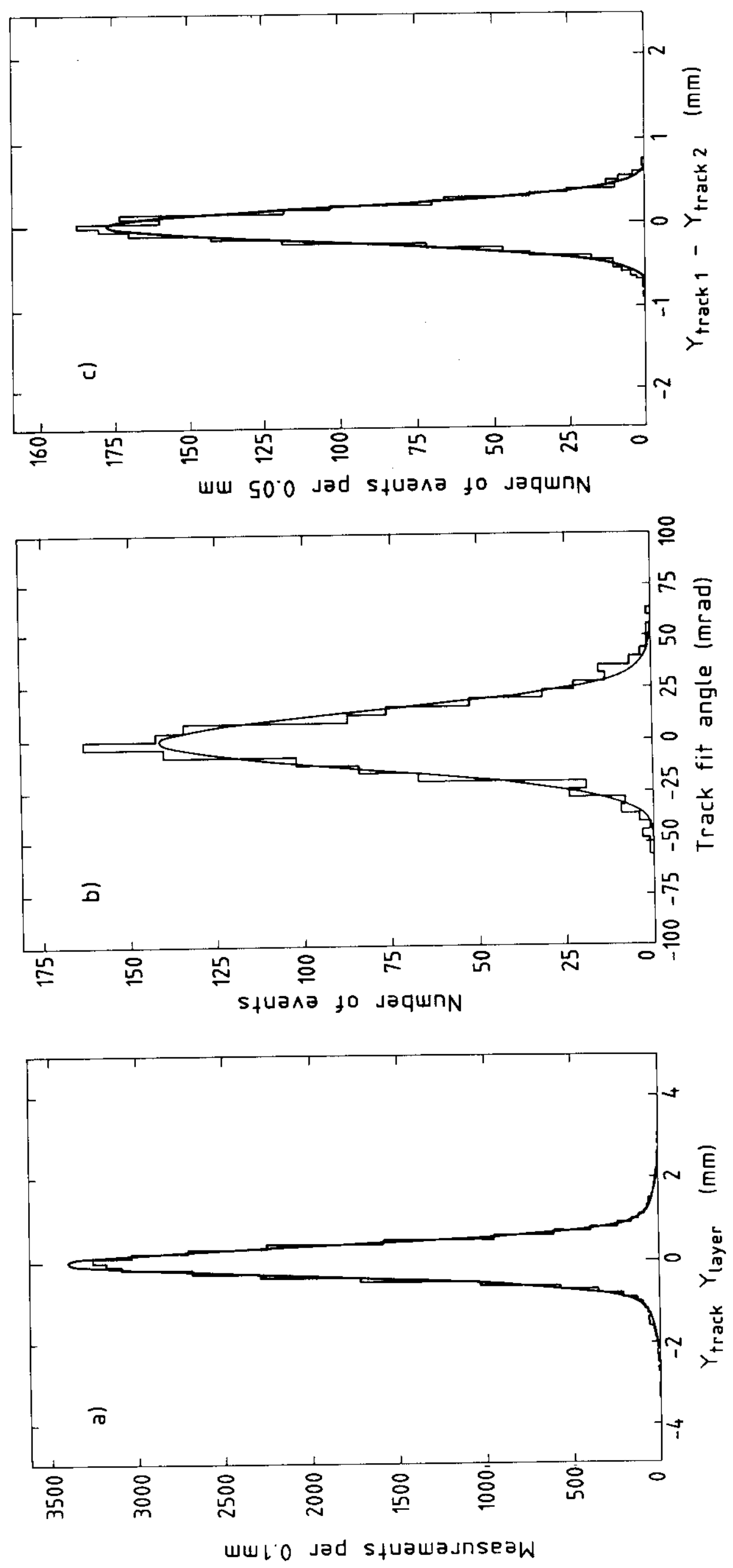


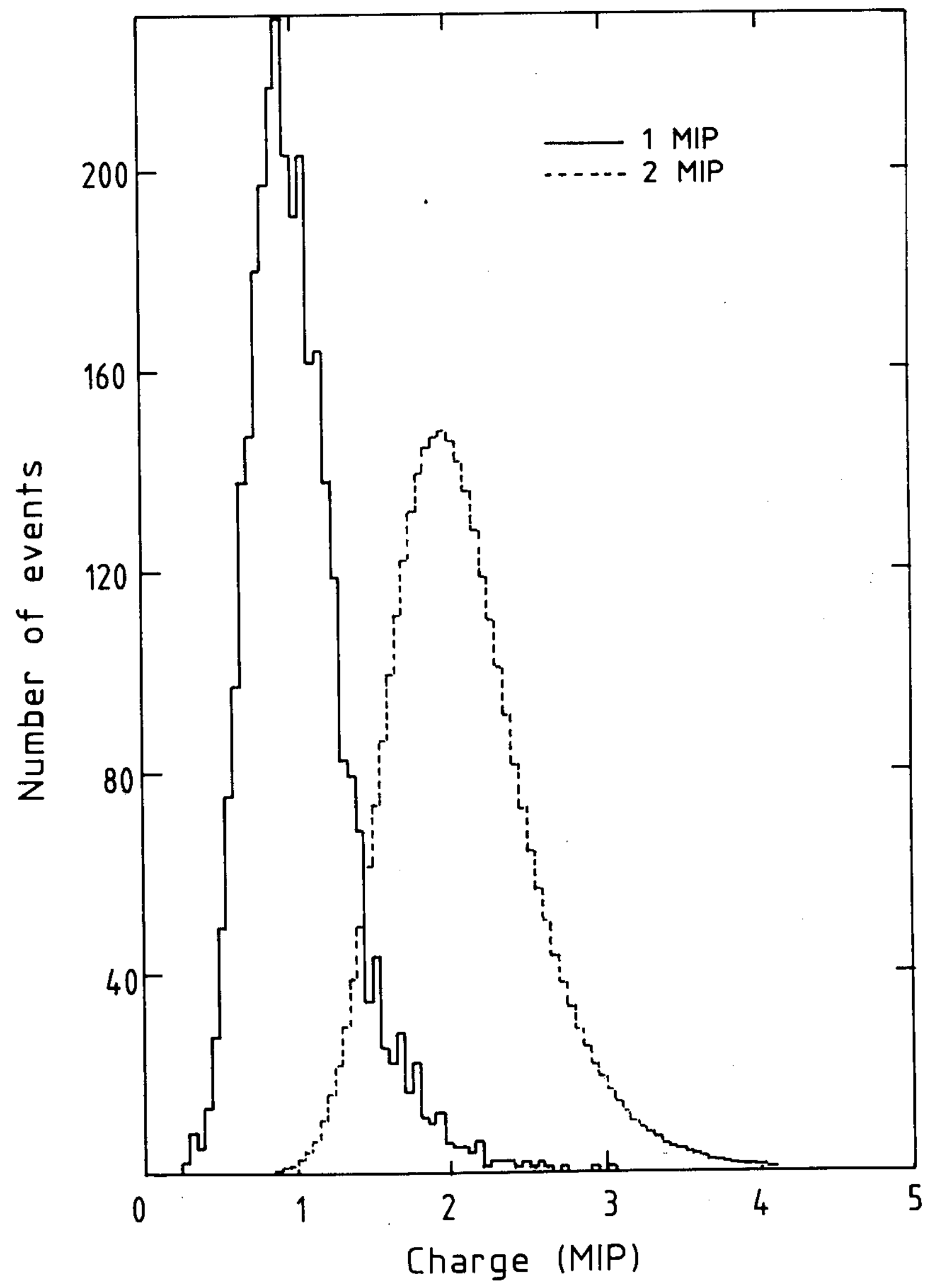

fig。 7 

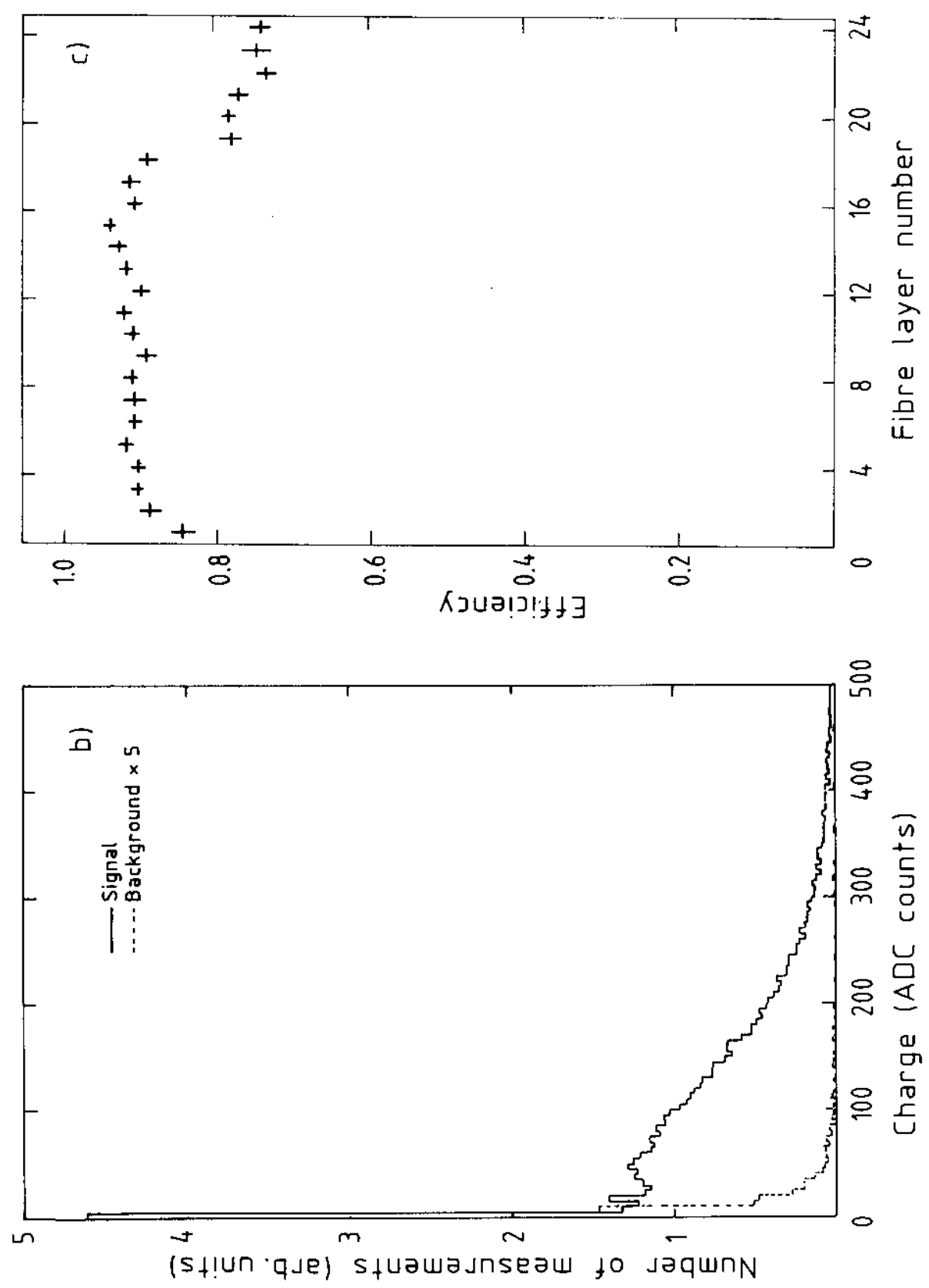

$\infty$
0
0
-4
4

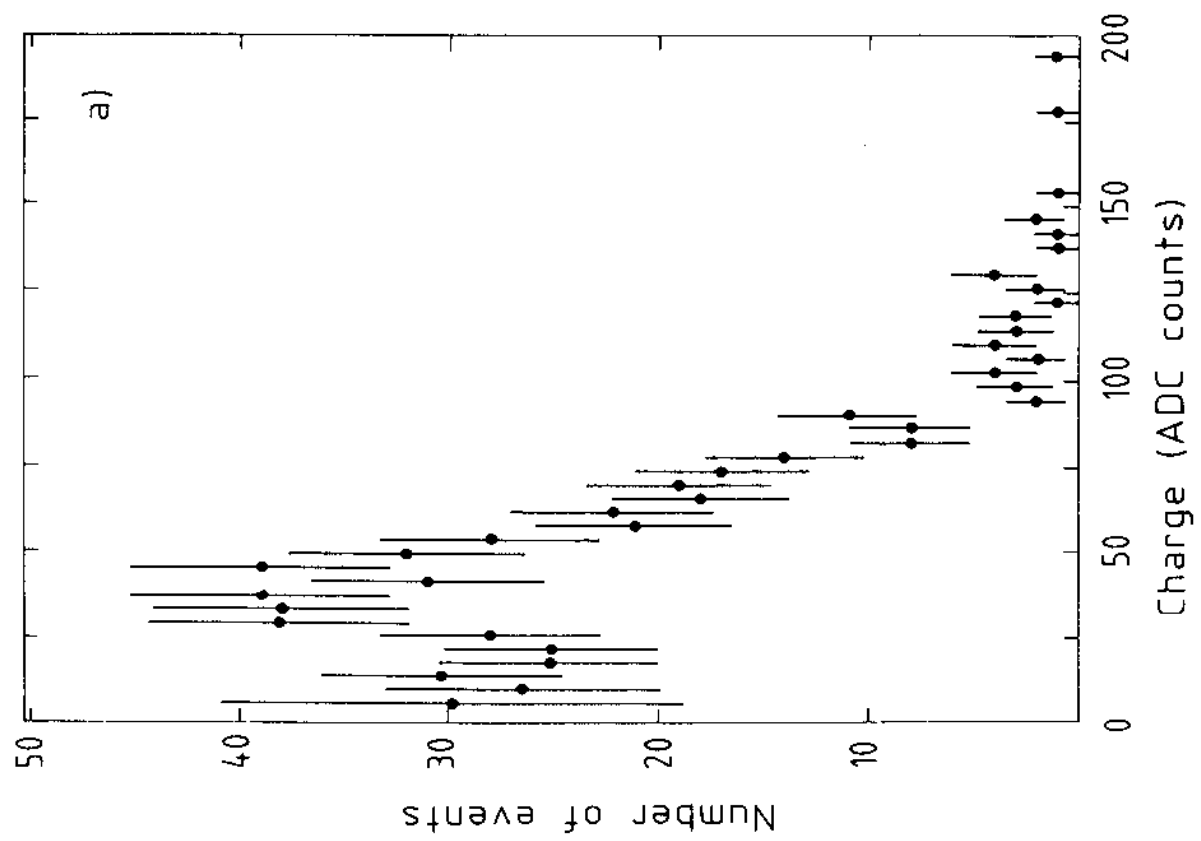




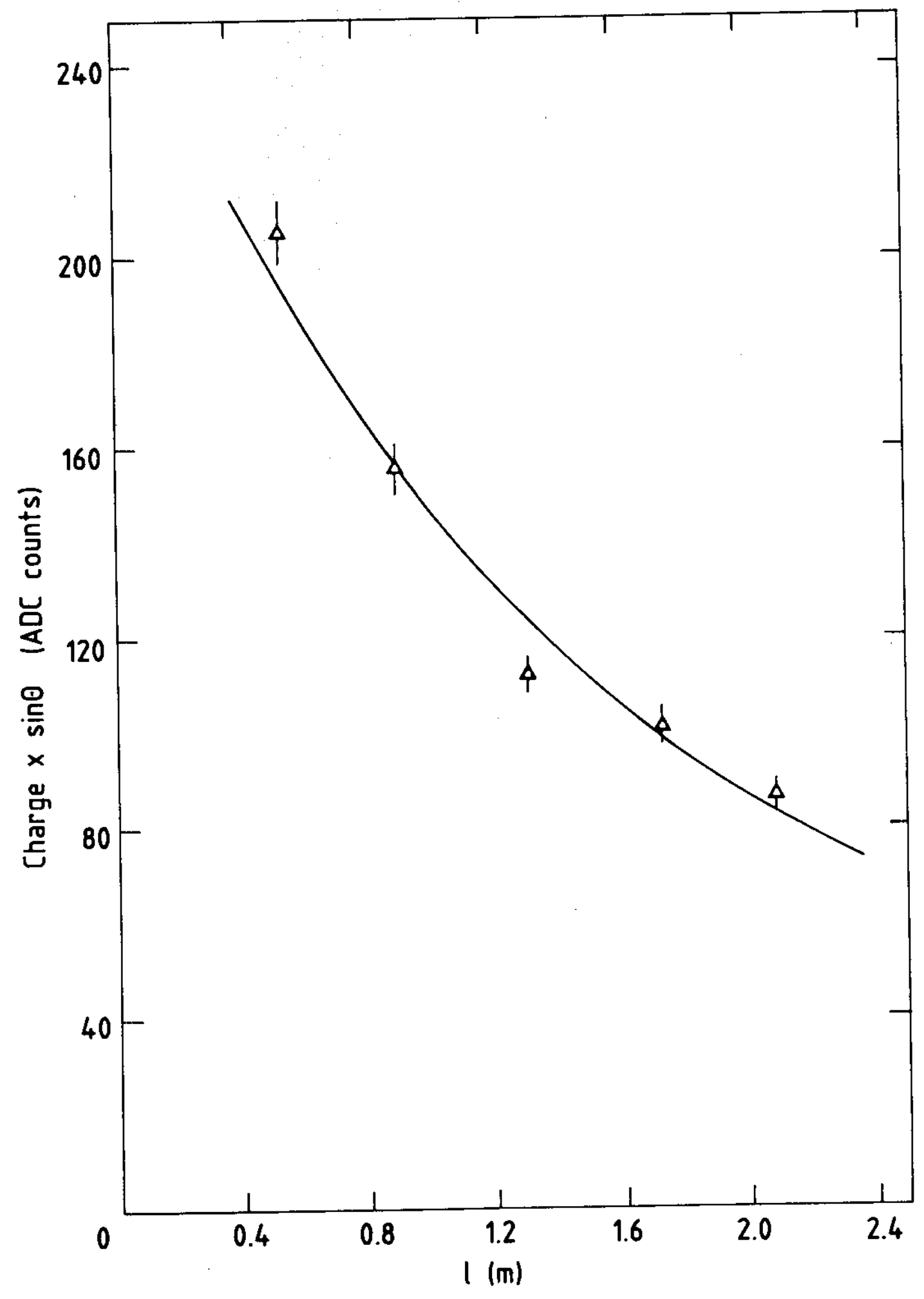

fig. 9 


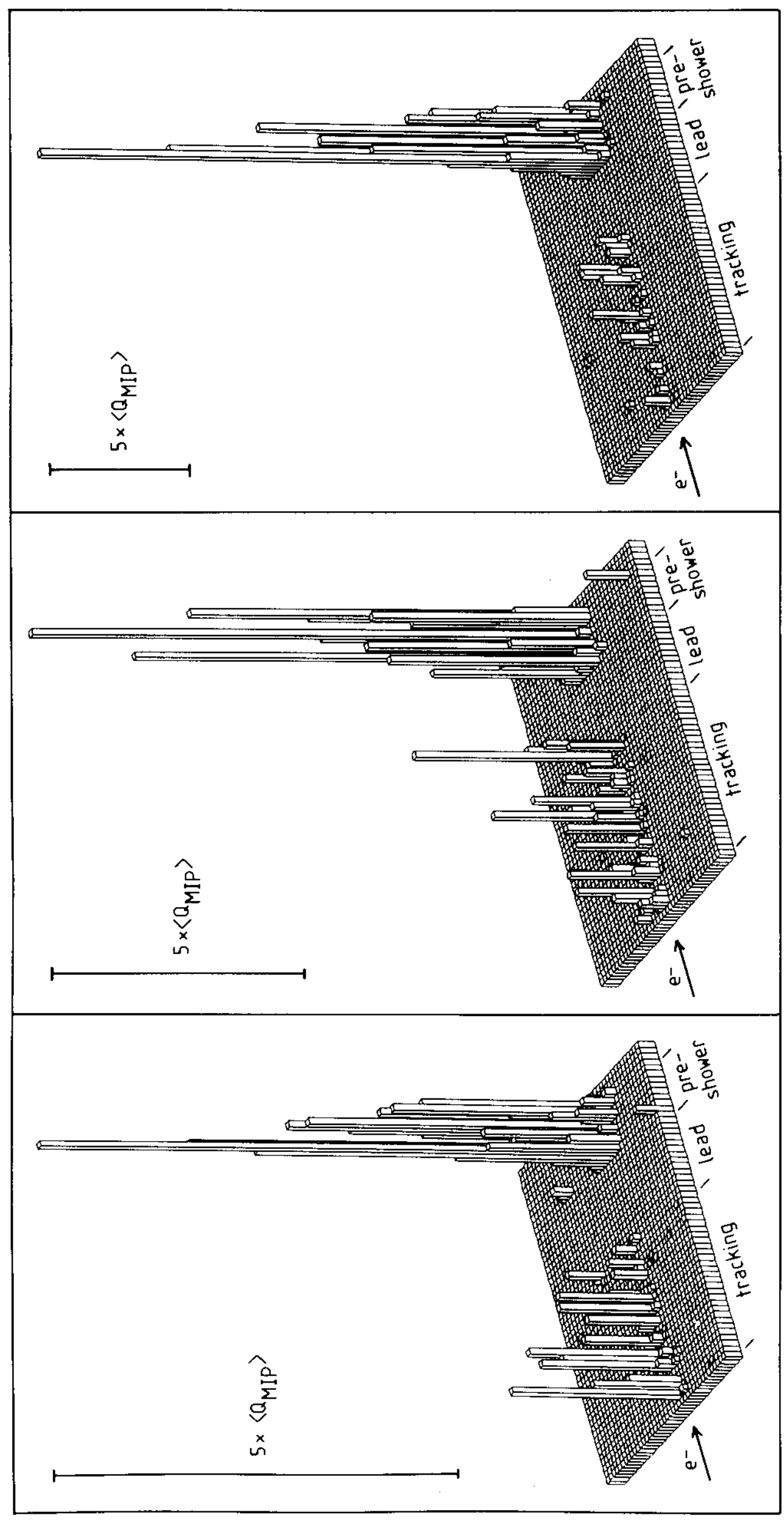

0
0
0
0
4 


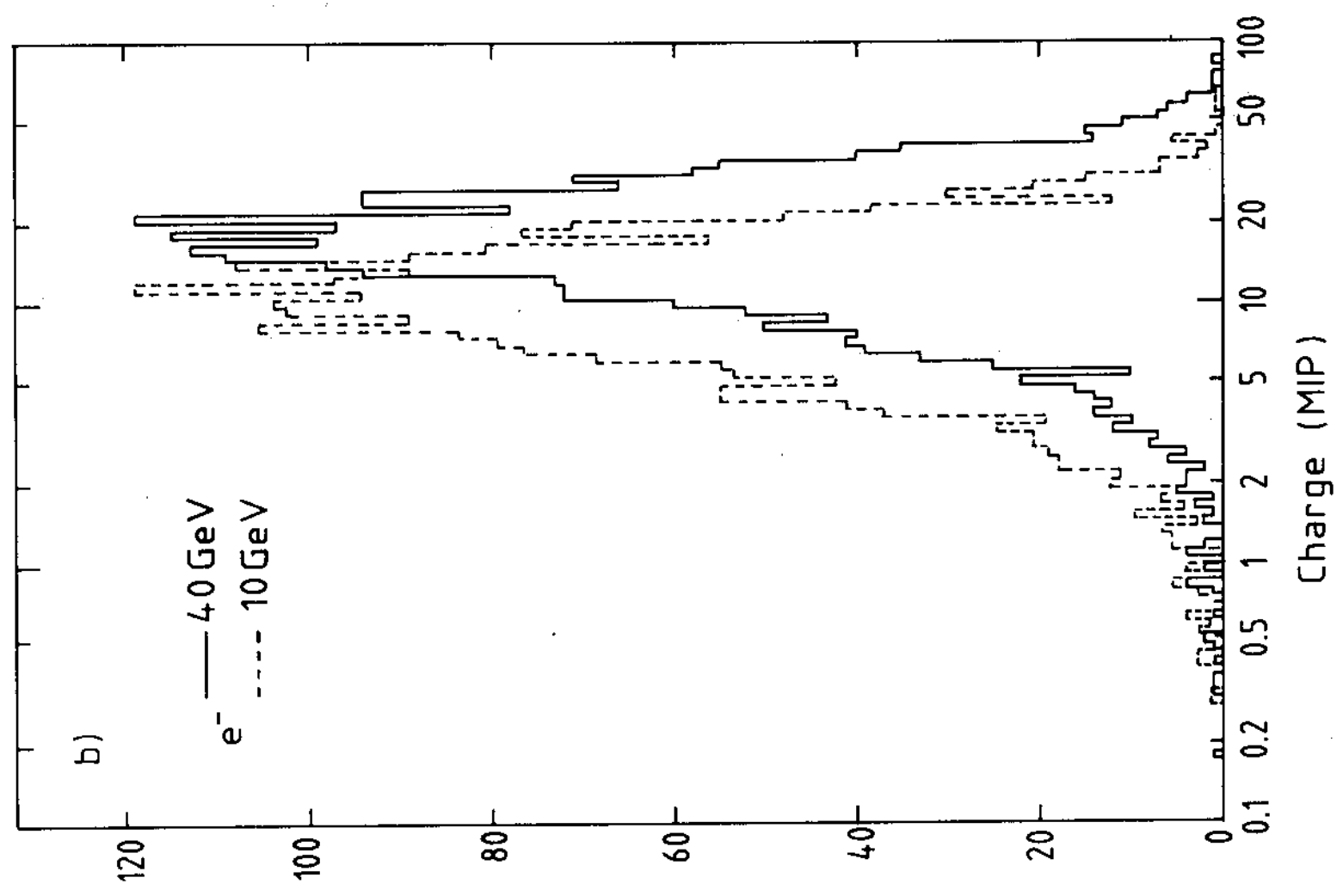

(stiun Kjejt!que) squana to jaqunN

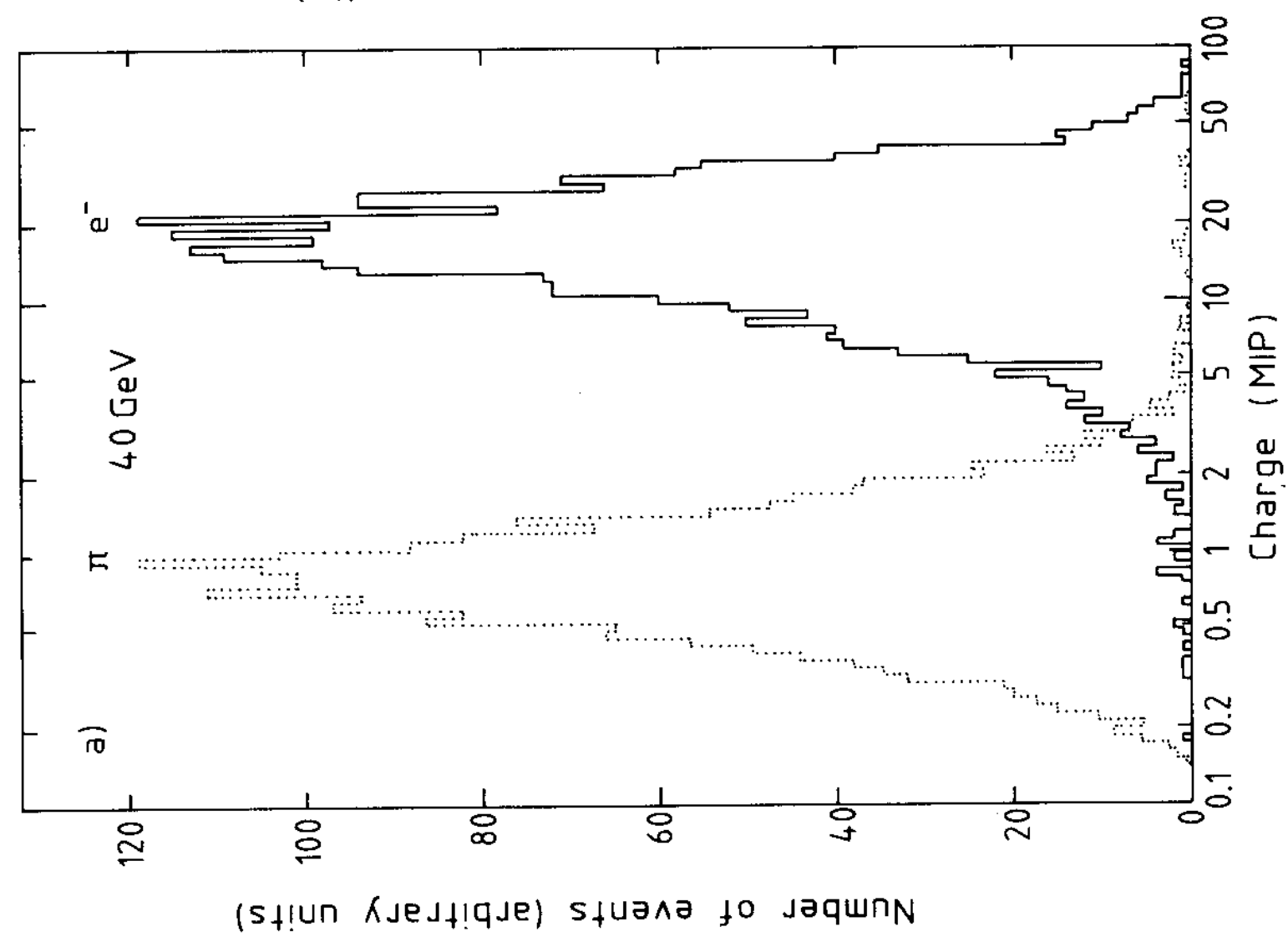




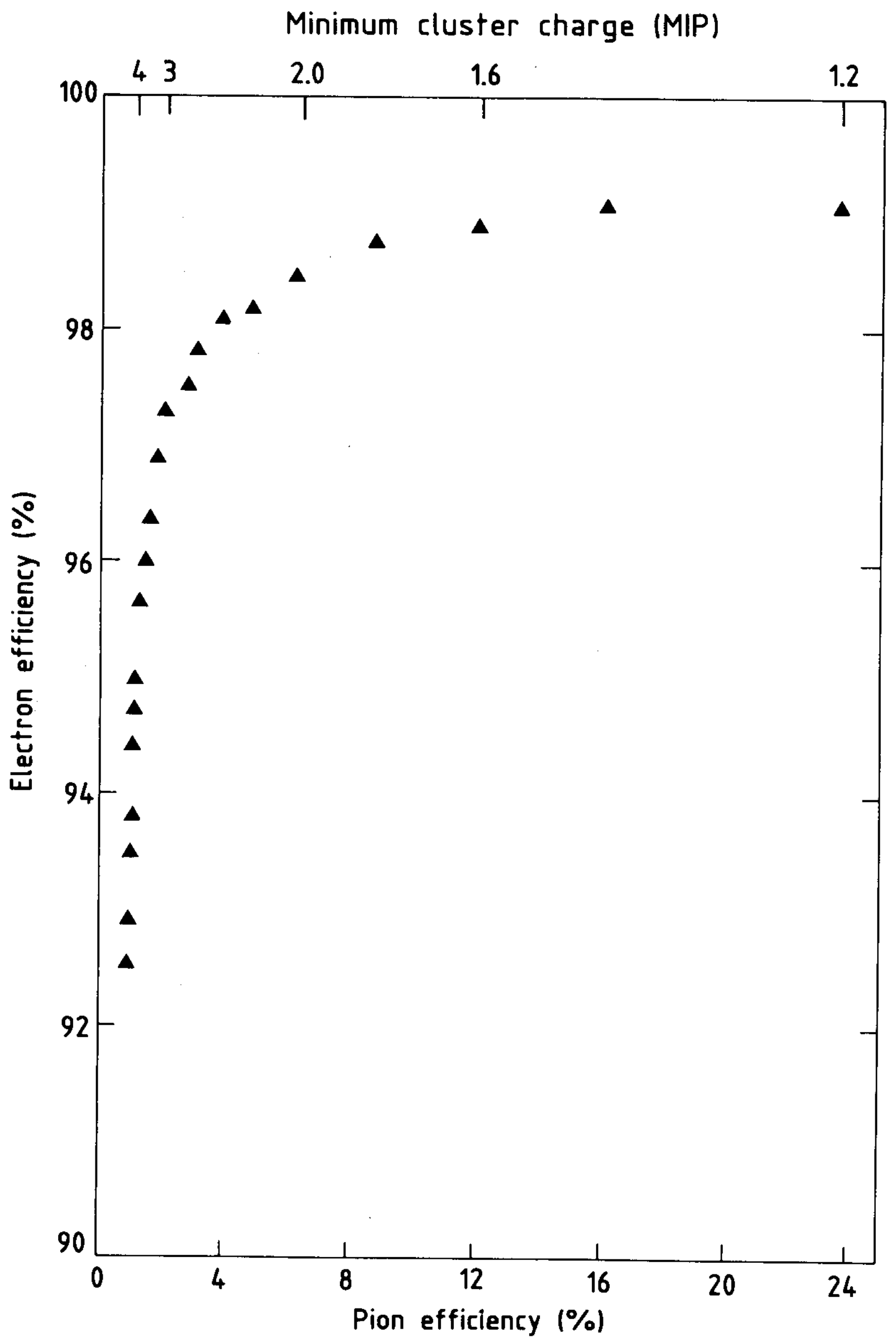

fig. 12 

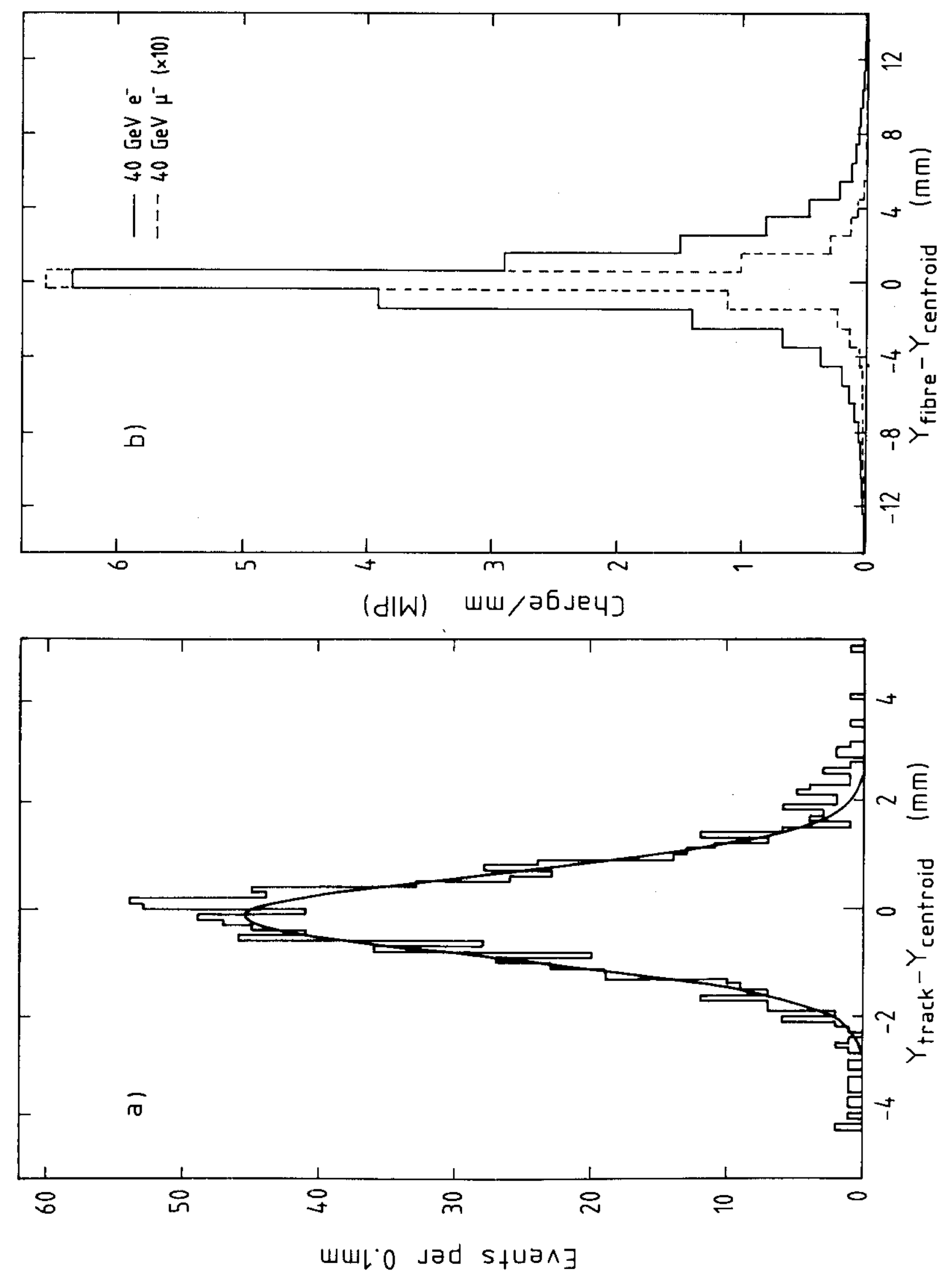


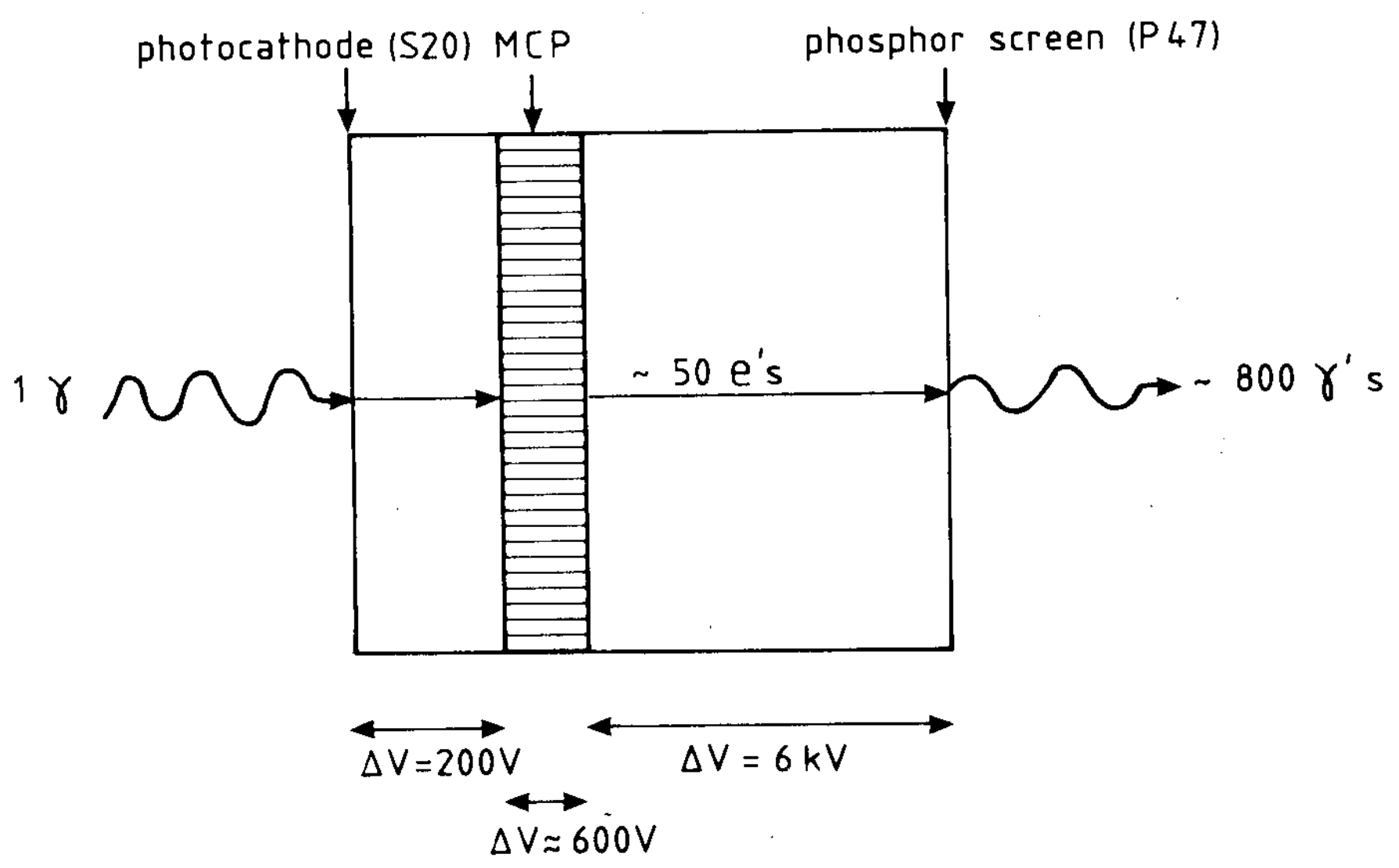

fig. 14 


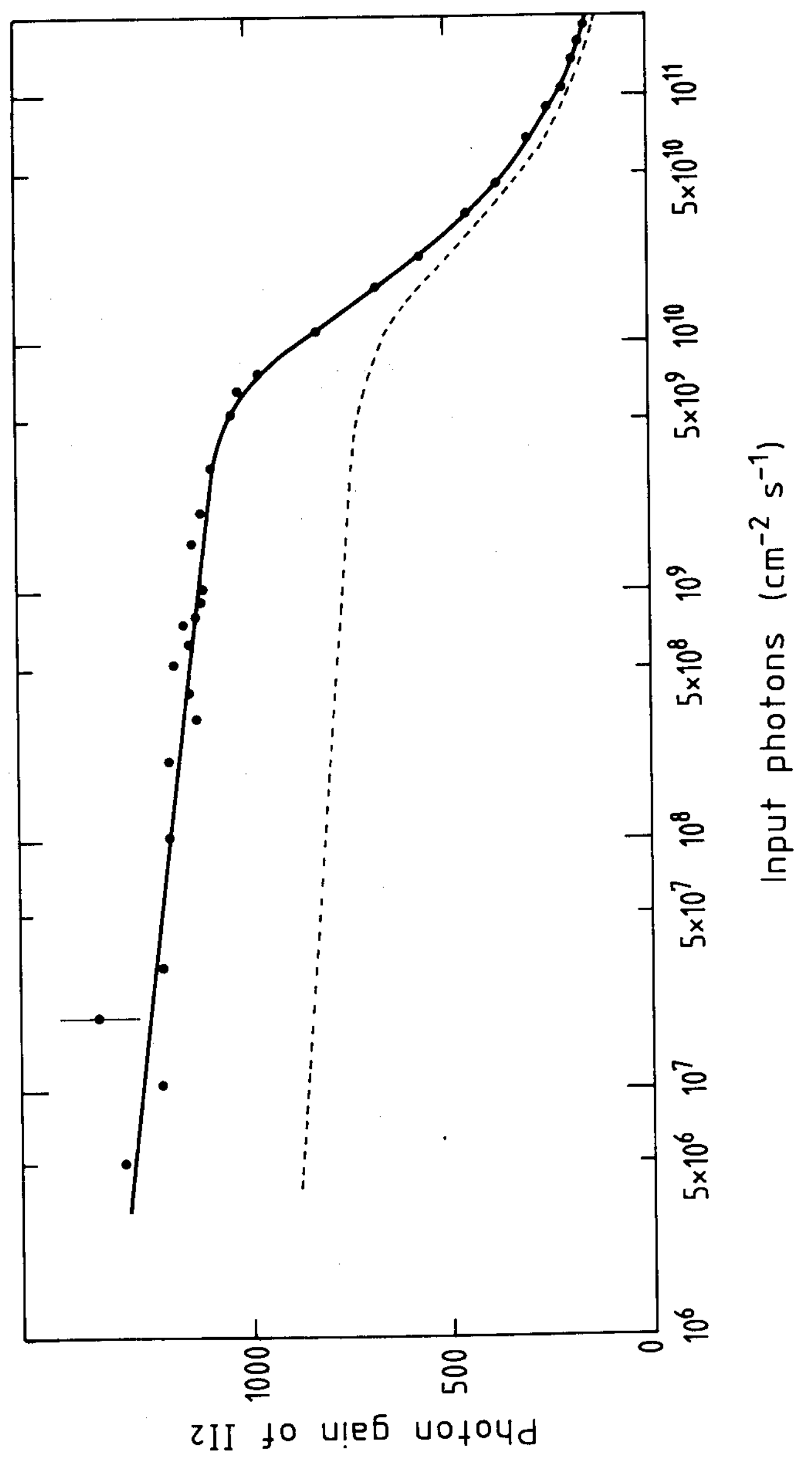




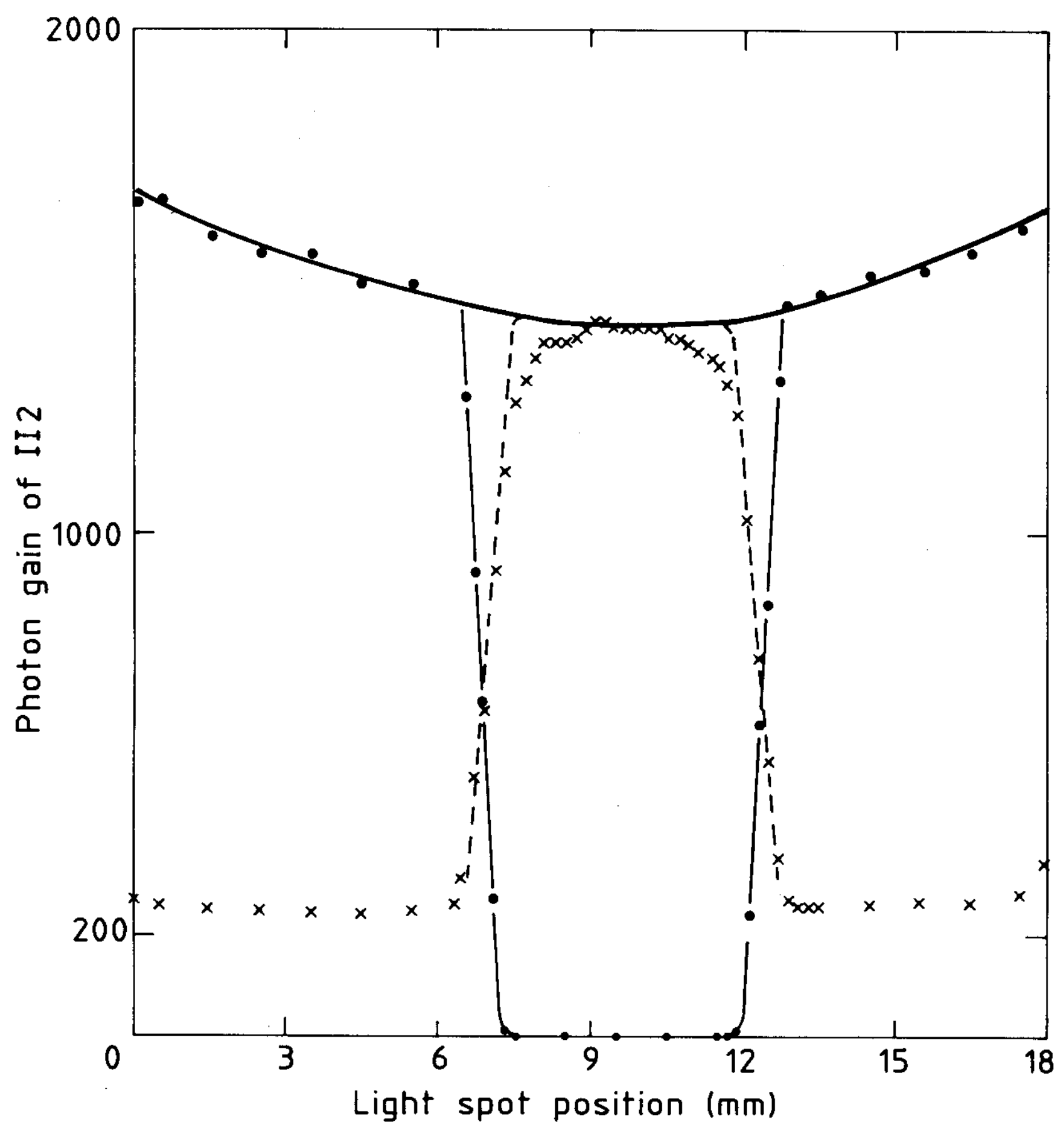

fig. 16 